\title{
Asymptotic approach on conjugate points for minimal time bang-bang controls
}

\author{
C. Silva ${ }^{a}$, E. Trélat ${ }^{b, *}$ \\ ${ }^{a}$ Department of Mathematics, University of Aveiro, Portugal and Math., Labo. MAPMO, Université d'Orléans, France \\ ${ }^{b}$ Université d'Orléans, UFR Sciences, Math., Labo. MAPMO, UMR 6628, Route de Chartres, BP 6759, 45067 Orléans cedex 2, France, tel. \\ (+33)(0)238492548, fax (+33)(0)238417205
}

\begin{abstract}
We focus on the minimal time control problem for single-input control-affine systems $\dot{x}=X(x)+u_{1} Y_{1}(x)$ in $\mathbb{R}^{n}$ with fixed initial and final time conditions $x(0)=\hat{x}_{0}, x\left(t_{f}\right)=\hat{x}_{1}$, and where the scalar control $u_{1}$ satisfies the constraint $\left|u_{1}(\cdot)\right| \leqslant 1$. For these systems a concept of conjugate time $t_{c}$ has been defined in e.g. $[3,30,33]$ in the bang-bang case. Besides, theoretical and practical issues for conjugate time theory are well known in the smooth case (see e.g. [5, 32]), and efficient implementation tools are available (see [11]). The first conjugate time along an extremal is the time at which the extremal loses its local optimality. In this work, we use the asymptotic approach developed in [44] and investigate the convergence properties of conjugate times. More precisely, for $\varepsilon>0$ small and arbitrary vector fields $Y_{1}, \ldots, Y_{m}$, we consider the minimal time problem for the control system $\dot{x}^{\varepsilon}=X\left(x^{\varepsilon}\right)+u_{1}^{\varepsilon} Y_{1}\left(x^{\varepsilon}\right)+\varepsilon \sum_{i=2}^{m} u_{i}^{\varepsilon} Y_{i}\left(x^{\varepsilon}\right)$, under the constraint $\sum_{i=1}^{m}\left(u_{i}^{\varepsilon}\right)^{2} \leqslant 1$, with the fixed boundary conditions $x^{\varepsilon}(0)=\hat{x}_{0}, x^{\varepsilon}\left(t_{f}\right)=\hat{x}_{1}$ of the initial problem. Under appropriate assumptions, the optimal controls of the latter regularized optimal control problem are smooth, and the computation of associated conjugate times $t_{c}^{\varepsilon}$ falls into the standard theory; our main result asserts the convergence, as $\varepsilon$ tends to 0 , of $t_{c}^{\varepsilon}$ towards the conjugate time $t_{c}$ of the initial bang-bang optimal control problem, as well as the convergence of the associated extremals. As a byproduct, we obtain an efficient algorithmic way to compute conjugate times in the bang-bang case.
\end{abstract}

Keywords:

optimal control, minimal time problem, bang-bang control, conjugate time

\section{Introduction}

\subsection{The optimal control problem}

Consider the single-input control-affine system in $\mathbb{R}^{n}$

$$
\dot{x}=X(x)+u_{1} Y_{1}(x)
$$

where $X$ and $Y_{1}$ are smooth vector fields, and the control $u_{1}$ is a measurable scalar function satisfying the constraint

$$
\left|u_{1}(\cdot)\right| \leqslant 1 .
$$

Let $\hat{x}_{0}$ and $\hat{x}_{1}$ be two points of $\mathbb{R}^{n}$. Assume that $\hat{x}_{1}$ is reachable from $\hat{x}_{0}$, that is, there exists a time $T>0$ and a control function $u_{1}(\cdot) \in L^{\infty}(0, T)$ satisfying the

\footnotetext{
${ }^{*}$ Corresponding author

Email addresses: cjoaosilva@ua.pt (C. Silva), emmanuel.trelat@univ-orleans.fr (E. Trélat )

${ }^{1} \mathrm{C}$. Silva is supported by FCT through the $\mathrm{PhD}$ fellowship SFRH/DB/27272/2006
}

Preprint submitted to Elsevier constraint (2), such that the trajectory $x(\cdot)$, solution of (1) with $x(0)=\hat{x}_{0}$, satisfies $x(T)=\hat{x}_{1}$.

We consider the optimal control problem (OCP) of determining a solution $\hat{x}(\cdot)$ associated to a control $\hat{u}_{1}(\cdot)$, on $\left[0, t_{f}\right]$, satisfying $(1)-(2)$ and steering $\hat{x}_{0}$ to $\hat{x}_{1}$ in minimal time $t_{f}$. We assume that such a solution $\hat{x}(\cdot)$ for (OCP) exists ${ }^{2}$.

According to the Pontryagin maximum principle (see [39]), there exists a non trivial absolutely continuous mapping $\hat{p}(\cdot):\left[0, t_{f}\right] \rightarrow \mathbb{R}^{n}$, called adjoint vector, and a real number $p^{0} \leqslant 0$, with $\left(\hat{p}(\cdot), p^{0}\right) \neq(0,0)$, such that

$$
\begin{aligned}
\dot{\hat{p}}(t) & =-\frac{\partial H}{\partial x}\left(\hat{x}(t), \hat{p}(t), p^{0}, \hat{u}_{1}(t)\right) \\
& =-\left\langle\hat{p}(t), \frac{\partial X}{\partial x}(\hat{x}(t))\right\rangle-\hat{u}_{1}(t)\left\langle\hat{p}(t), \frac{\partial Y_{1}}{\partial x}(\hat{x}(t))\right\rangle
\end{aligned}
$$

where the function

$$
H\left(x, p, p^{0}, u_{1}\right)=\left\langle p, X(x)+u_{1} Y_{1}(x)\right\rangle+p^{0}
$$

\footnotetext{
${ }^{2}$ See e.g. [14] for existence results of optimal solutions.
} 
is called the Hamiltonian, and the maximization condition

$$
H\left(\hat{x}(t), \hat{p}(t), p^{0}, \hat{u}_{1}(t)\right)=\max _{|w| \leqslant 1} H\left(\hat{x}(t), \hat{p}(t), p^{0}, w\right)
$$

holds almost everywhere on $\left[0, t_{f}\right]$. Moreover, $\max _{|w| \leqslant 1} H\left(\hat{x}(t), \hat{p}(t), p^{0}, w\right)=0$ for every $t \in\left[0, t_{f}\right]$. The quadruple $\left(\hat{x}(\cdot), \hat{p}(\cdot), p^{0}, \hat{u}_{1}(\cdot)\right)$ is called an extremal. The extremal is said normal whenever $p^{0} \neq 0$, and in that case it is usual to normalize the adjoint vector so that $p^{0}=-1$; otherwise it is said abnormal. It follows from (4) that

$$
\hat{u}_{1}(t)=\operatorname{sign}\left\langle\hat{p}(t), Y_{1}(\hat{x}(t))\right\rangle
$$

for almost every $t$, provided that the (continuous) switching function

$$
\varphi_{1}(t)=\left\langle\hat{p}(t), Y_{1}(\hat{x}(t))\right\rangle
$$

does not vanish on any subinterval of $\left[0, t_{f}\right]^{3}$. Such an extremal $\left(\hat{x}(\cdot), \hat{p}(\cdot), p^{0}, \hat{u}_{1}(\cdot)\right)$ is then completely determined by the initial adjoint vector $\hat{p}(0)$. This extremal is a priori defined on the time interval $\left[0, t_{f}\right]$, but since it is completely determined by the differential system (1)-(3) and its initial condition, it may be extended forward on a maximal time interval $I$ of $[0,+\infty)$, containing $\left[0, t_{f}\right]$. In this way, we consider the trajectory $\hat{x}(\cdot)$ on this maximal interval $I$.

Note that, since $\hat{x}(\cdot)$ is optimal on $\left[0, t_{f}\right]$, and since the control system under study is autonomous, it follows that $\hat{x}(\cdot)$ is as well optimal for the problem of steering the system (1) from $\hat{x}(0)=\hat{x}_{0}$ to $\hat{x}(t)$, for every $t \in$ $\left(0, t_{f}\right]$.

We assume that the extremal $\left(\hat{x}(\cdot), \hat{p}(\cdot), p^{0}, \hat{u}_{1}(\cdot)\right)$ is bang-bang on the interval $I$, that is, the switching function $\varphi_{1}$ does not vanish on any subinterval of $I$. Denote by $\hat{\tau}_{1}, \ldots, \hat{\tau}_{s}, \ldots$ the zeros of $\varphi_{1}$ on $I$ (possibly in infinite number), called switching times, such that $0<\hat{\tau}_{1}<\ldots<\hat{\tau}_{s} \ldots$ As explained above, there holds

$$
\hat{u}_{1}(t)=\left\{\begin{array}{rcc}
1 & \text { if } & \varphi_{1}(t)>0, \\
-1 & \text { if } & \varphi_{1}(t)<0,
\end{array}\right.
$$

for every $t \in I$.

We assume moreover that the extremal $\left(\hat{x}(\cdot), \hat{p}(\cdot), p^{0}, \hat{u}_{1}(\cdot)\right) \quad$ satisfies the strict bang-bang Legendre condition, that is,

$$
\dot{\varphi}_{1}\left(\hat{\tau}_{j}\right)=\left.\frac{d}{d t}\left\langle\hat{p}(t), Y_{1}(\hat{x}(t))\right\rangle\right|_{t=\hat{\tau}_{j}} \neq 0,
$$

\footnotetext{
${ }^{3}$ The case where the switching function may vanish on a subinterval is related to singular trajectories, and is outside of the scope of this article where we focus on the bang-bang case.
}

for every switching time. This condition implies that the switching times are isolated, and moreover are in finite number on every compact subinterval of $I$. In particular, we assume that there are exactly $s$ switching times on the interval $\left[0, t_{f}\right]$, such that $0<\hat{\tau}_{1}<\ldots<\hat{\tau}_{s}<t_{f}$.

Definition 1.1. Let $T>0, T \in I$. The trajectory $\hat{x}(\cdot)$ is said to be locally minimal time on $[0, T]$ in $C^{0}$ topology if there exist a neighborhood $W$ of the trajectory $\hat{x}(\cdot)$ in $\mathbb{R}^{n}$ and a real number $\eta>0$ such that, for every trajectory $y(\cdot)$ that is solution of (1), contained in $W$, associated with a control $v$ on $[0, T+\eta]$ satisfying the constraint (2), satisfying $y(0)=\hat{x}_{0}$ and $y\left(t_{1}\right)=\hat{x}(T)$ with $t_{1} \in[0, T+\eta]$, there holds $t_{1} \geqslant T$.

The $C^{0}$ local optimality is also called strong local optimality. The notion of global optimality is defined similarly, with $W=\mathbb{R}^{n}$ and $\eta=+\infty$.

The Pontryagin maximum principle mentioned formerly is a necessary first order condition for optimality; conversely, extremals are not necessarily locally optimal, and there have been many works on high-order necessary optimality conditions (see e.g. [12]) and on sufficient (first and second order) optimality conditions detailed in the next section.

\subsection{Second order optimality conditions and conjugate times for bang-bang controls}

Consider the extremal $\left(\hat{x}(\cdot), \hat{p}(\cdot), p^{0}, \hat{u}_{1}(\cdot)\right)$ of the problem (OCP) introduced previously.

Definition 1.2. The cut time $t_{\text {cut }}\left(\hat{x}_{0}\right)$ is defined as the first positive time of $I$ beyond which the trajectory $\hat{x}(\cdot)$ loses its global optimality status for the problem of steering the system (1)-(2) from $\hat{x}_{0}$ to $\hat{x}_{1}$ in minimal time, with the agreement that $t_{\text {cut }}\left(\hat{x}_{0}\right)=+\infty$ whenever $\hat{x}(\cdot)$ is globally optimal on every interval $[0, T], T>0$, $T \in I$. The point $\hat{x}\left(t_{\text {cut }}\left(\hat{x}_{0}\right)\right)$ is called a cut point.

Whereas such a global optimality status is difficult to characterize, the local optimality status of a trajectory may be characterized using the concept of conjugate time, that is, the time at which the optimal trajectory $\hat{x}(\cdot)$ loses its local optimality. We next recall well known facts on first conjugate times of solutions $\hat{x}(\cdot)$ of the optimal control problem (OCP) associated to bangbang controls $\hat{u}_{1}(\cdot)$.

The definition and computation of conjugate points are an important topic in the theory of calculus of variations (see e.g. [9]). In [42] the investigation of the definition and computation of conjugate points for minimal time control problems is based on the study of second order conditions. In fact, second order necessary 
and/or sufficient conditions are crucial for study of the first conjugate time of the problem (OCP). In [46], the theory of envelopes and conjugate points is used for the study of the structure of locally optimal bang-bang trajectories for the problem (OCP) in $\mathbb{R}^{2}$ and $\mathbb{R}^{3}$; these results were generalized in [19].

Second order optimality conditions. When the optimal control problem has a nonlinear control system and the extremal controls are continuous, the literature on first and/or second order sufficient conditions is vast, see e.g. [10, 16, 21, 24, 26, 28, 31, 32, 49] and references therein. In this case numerical procedures are available to test second order sufficient conditions, see e.g. $[8,23,26]$. For second order necessary and/or sufficient conditions of optimal control problems with nonlinear control systems and discontinuous controls see e.g. [37] and references therein.

We will next focus on second order necessary and/or sufficient optimality conditions for optimal control problems with affine-control systems and bang-bang optimal controls.

In [43] a minimal time control problem for affinecontrol systems is considered and first and second order sufficient optimality conditions are derived, for bangbang Pontryagin extremal controls which are $L^{1}$-locally optimal. In [30] the same optimal control problem is studied and the authors provide sufficient conditions for strong local optimality and develop numerical methods to test the positive definiteness of a specific quadratic form. In both papers [43] and [30], the sufficient optimality conditions are expressed in terms of quadratic forms, however although the same critical subspace is used, the quadratic form in [43] is a lower bound for the one in [30]. In fact, the second order sufficient optimality condition in [30] is always fulfilled whenever the corresponding condition in [43] is.

In $[27,30]$ optimization methods are given to test second order sufficient optimality conditions for optimal control problems with bounded scalar controls [30], and vector-valued controls [27].

In [3] the authors derive second order sufficient conditions, under the same regularity assumptions as [30], for an optimal control problem in the Mayer form with fixed final time, with affine-control systems and bangbang optimal controls. In [35] the authors showed that, in certain cases, the second order sufficient conditions given in [30] are equivalent to the ones in [3]. In the cases where the equivalence holds, the results obtained in [35] extend those in [3] to the problem of free final time, with mixed initial and terminal conditions of equality and inequality type. The detailed proofs of the main results in [35] are given in [36]. In [3] a finitedimensional subproblem is considered which consists in moving the switching times and a second variation is defined as a certain quadratic form associated to this subproblem; then, finding a conjugate time consists in testing the positivity of that quadratic form. The authors prove that this can only happen at a switching time.

In [38] the minimal time problem for control affine systems is studied. An analogous quadratic form to the one in [3] is defined, but the kind of optimality studied is a stronger one (state local optimality).

Quadratic forms. As mentioned above the quadratic forms defined in $[3,30]$ are equivalent (see $[35,36]$ ), although the way they are defined is different. We only give a brief sketch of a possible procedure to define the quadratic form.

Let $F\left(t ; \tau_{1}, \ldots, \tau_{s}\right)=x\left(t ; \tau_{1}, \ldots, \tau_{s}\right)$ be the mapping associated with the finite-dimensional problem associated to (OCP) that consists in moving the switching times $\tau_{1}, \ldots, \tau_{s}$ in a neighborhood of the reference switching times $\hat{\tau}_{1}, \ldots, \hat{\tau}_{s}$ (see $[3,27,35,36,38]$ ), where $x\left(t ; \tau_{1}, \ldots, \tau_{s}\right)$ is the trajectory solution of $(1)$, on $[0, t]$, with $x(0)=\hat{x}_{0}$, associated to the bang-bang control $u_{1}(\cdot)$ with switching times $\tau_{1}, \ldots, \tau_{s}$ and such that it coincides with the reference trajectory $\hat{x}(\cdot)$ whenever $\tau_{i}=\hat{\tau}_{i}$ for every $i$. Note that the trajectory $x\left(\cdot ; \tau_{1}, \ldots, \tau_{s}\right)$ is not the projection of an extremal whenever $\tau_{i} \neq \hat{\tau}_{i}$. The mapping $F$ is well defined for $t$ in a neighborhood of $t_{f}$ and $\tau_{i}$ in a neighborhood of $\hat{\tau}_{i}$ for every $i$, and is the composition of smooth mappings, therefore is differentiable. Denoting $\tau=\left(\tau_{1}, \ldots, \tau_{s}\right)$, one has

$$
\frac{\partial F}{\partial \tau}\left(t ; \tau_{1}, \ldots, \tau_{s}\right)=\left(\begin{array}{ccc}
\frac{\partial x_{1}}{\partial \tau_{1}}(\cdot) & \ldots & \frac{\partial x_{1}}{\partial \tau_{s}}(\cdot) \\
\vdots & \vdots & \vdots \\
\frac{\partial x_{n}}{\partial \tau_{1}}(\cdot) & \ldots & \frac{\partial x_{n}}{\partial \tau_{s}}(\cdot)
\end{array}\right),
$$

and

$$
\frac{\partial F}{\partial t}\left(t ; \tau_{1}, \ldots, \tau_{s}\right)=\dot{x}\left(t ; \tau_{1}, \ldots, \tau_{s}\right)
$$

Since $\hat{x}(\cdot)$ is optimal, it follows that

$$
\operatorname{rank}\left(\frac{\partial F}{\partial \tau}\left(t ; \hat{\tau}_{1}, \ldots, \hat{\tau}_{s}\right)\right) \leqslant n-1 .
$$

Indeed, otherwise, if $\operatorname{rank}\left(\frac{\partial F}{\partial \tau}\left(t ; \hat{\tau}_{1}, \ldots, \hat{\tau}_{s}\right)\right)=n$ then $F$ would be a local submersion, which contradicts the optimality of $\hat{x}(\cdot)$. Therefore, there exists a multiplier $\psi_{t} \in \mathbb{R}^{n} \backslash\{0\}$ such that $\psi_{t} \cdot \frac{\partial F}{\partial \tau}\left(t ; \hat{\tau}_{1}, \ldots, \hat{\tau}_{s}\right)=0$. Denote by $Q_{t}$ the intrinsic second derivative of the mapping $F$, defined by

$$
Q_{t}=\left.\psi_{t} \cdot \frac{\partial^{2} F}{\partial \tau^{2}}\left(t ; \hat{\tau}_{1}, \ldots, \hat{\tau}_{s}\right)\right|_{\operatorname{ker} \frac{\partial F}{\partial \tau}\left(t ; \hat{\tau}_{1}, \ldots, \hat{\tau}_{s}\right)} .
$$


Explicit formulas of $Q_{t}$ are given in [3, 4, 30, 38]; in particular formulas in terms of Lie brackets of the vector fields can be derived.

The next theorem, combination of several known results, provides a necessary and/or sufficient condition for strong local optimality.

Theorem $1([3,4,30,33,38])$. Let $\left(\hat{x}(\cdot), \hat{p}(\cdot), p^{0}, \hat{u}_{1}(\cdot)\right)$ be a bang-bang extremal for $(\boldsymbol{O C P})$ defined on a maximal time interval I of $[0,+\infty)$ containing $\left[0, t_{f}\right]$. If this extremal satisfies the strict bang-bang Legendre condition on I, then for every $t \in I$, the following holds:

- If the quadratic form $Q_{t}$ is positive definite then $\hat{x}(\cdot)$ is a local minimizer in the $C^{0}$ topology on $[0, t]$.

- Assume moreover that $\hat{x}(\cdot)$ has a unique extremal lift (up to a multiplicative scalar) $\left(\hat{x}(\cdot), \hat{p}(\cdot), p^{0}, \hat{u}_{1}(\cdot)\right)$, which is moreover normal $\left(p^{0}=-1\right)$. If $\hat{x}(\cdot)$ is locally optimal in the $C^{0}$ topology on $[0, t]$ then $Q_{t}$ is nonnegative.

Remark 1.1. Under the assumptions of the Theorem 1, the set

$$
\left\{t>0 \mid Q_{t} \text { has a nontrivial kernel }\right\}
$$

is discrete and can only consist of some switching times (see [3]). This remark permits to define the notion of first conjugate time.

Definition 1.3. The first conjugate time $t_{c}$ of $\hat{x}(\cdot)$ is defined by

$t_{c}=\sup \left\{t \mid Q_{t}\right.$ is positive definite $\}=\inf \left\{t \mid Q_{t}\right.$ is indefinite $\}$.

The point $\hat{x}\left(t_{c}\right)$ is called the first conjugate point of the trajectory $\hat{x}(\cdot)$.

Remark 1.2. A conjugate time can only occur at a switching time.

Extremal field approach. Sufficient optimality conditions for a general optimal control problem are provided in [33] (see also [3, 38]) with a different point of view than the one recalled in the previous paragraph. In [33] the authors study local optimality conditions for both continuous and piecewise continuous (including bangbang) controls. The sufficient conditions developed in that article are based on the method of characteristics and the theory of extremal fields. Sufficient optimality conditions are given for embedding a reference trajectory into a local field of broken extremals ${ }^{4}$. The

\footnotetext{
${ }^{4}$ Broken extremals are associated to piecewise continuous controls.
}

occurrence of a conjugate point is related with a socalled overlap of the flow near the switching surface. Second order sufficient optimality conditions stated in [33] have been tested numerically for bang-bang control problems, see e.g. [20]. See also [47] where sufficient optimality conditions for bang-bang controls based on the extremal field approach are studied.

In $[1,2,3]$, using Hamiltonian methods and the extremal field theory, the authors construct, under certain conditions, a non-intersecting field of state extremals ${ }^{5}$ that covers a given extremal trajectory $\hat{x}(\cdot)$. In [3] the authors associate the occurrence of a conjugate point with a fold point of the flow of the extremal field. We next recall the Hamiltonian approach presented in $[3,38]$.

For every $z_{0}=\left(x_{0}, p_{0}\right) \in \mathbb{R}^{n} \times \mathbb{R}^{n}$, let $z\left(\cdot, z_{0}\right)=$ $\left(x\left(\cdot, z_{0}\right), p\left(\cdot, z_{0}\right)\right)$ denote the solution of the system of equations (1) and (3), with the control (5), such that $z\left(0, z_{0}\right)=z_{0}$. The exponential mapping is then defined by

$$
\exp \left(t, z_{0}\right)=x\left(t, z_{0}\right) .
$$

In (OCP) as in the problems considered in [3] and [38] the initial point is not free $\left(\hat{x}_{0}\right.$ is a fixed point of $\left.\mathbb{R}^{n}\right)$. To apply the Hamiltonian approach presented in [3, 38], we consider a $C^{2}$ function $\alpha: \mathbb{R}^{n} \rightarrow \mathbb{R}$ such that $\alpha^{\prime}\left(\hat{x}_{0}\right)=\hat{p}_{0}$, where $\alpha^{\prime}\left(x_{0}\right)$ denotes $\frac{\partial \alpha}{\partial x}\left(x_{0}\right)$ and $\hat{p}_{0}=\hat{p}(0)$. The function $\alpha$ represents a penalization on the initial point $\hat{x}_{0}$ and a new finite-dimensional subproblem is considered, with free initial point $\alpha\left(\hat{x}_{0}\right)$, that consists in moving the switching times and minimizing $\alpha\left(\hat{x}_{0}\right)+t_{f}$.

The existence of a function $\alpha$ in the previous conditions was proved in [17]. Moreover, in [38] the authors proved that if the quadratic form (6) is positive definite, then the quadratic form associated to the finitedimensional subproblem of moving the switching times with free initial point is also positive definite.

Let $O$ be a neighborhood of the initial point $\hat{x}_{0}$. Let $x_{0} \in O$; define the switching time functions $\tau_{j}: O \rightarrow \mathbb{R}$ with

$$
\tau_{0}\left(x_{0}\right)=0 \text { and } \tau_{j}\left(\hat{x}_{0}\right)=\hat{\tau}_{j}, \quad j=1, \ldots, s,
$$

such that, for $j=1, \ldots, s$,

$$
\begin{aligned}
& \varphi_{1}\left(\tau_{j}\left(x_{0}\right)\right)= \\
& \quad\left\langle p\left(\tau_{j}\left(x_{0}\right), x_{0}, \alpha^{\prime}\left(x_{0}\right)\right), Y_{1}\left(x\left(\tau_{j}\left(x_{0}\right), x_{0}, \alpha^{\prime}\left(x_{0}\right)\right)\right)\right\rangle=0 .
\end{aligned}
$$

\footnotetext{
${ }^{5}$ By non-intersecting extremals we mean that for any fixed $t \in$ $\left(0, t_{c}\right)$ and any extremal trajectories $x(\cdot), y(\cdot)$ with initial points $x_{0}, y_{0}$, respectively, with $x_{0}, y_{0}$ close to $\hat{x}_{0}$, we have $x(t) \neq y(t)$.
} 
In other words, $\tau_{j}\left(x_{0}\right)$ is the $j^{\text {th }}$-switching time of the extremal $x\left(\cdot, x_{0}, \alpha^{\prime}\left(x_{0}\right)\right), p\left(\cdot, x_{0}, \alpha^{\prime}\left(x_{0}\right)\right)$ starting from $\left(x_{0}, \alpha^{\prime}\left(x_{0}\right)\right)$, with $x_{0}$ close to $\hat{x}_{0}$.

Since $\hat{x}(\cdot)$ is a minimal time trajectory, there holds $\max _{|w| \leq 1} H\left(\hat{x}_{0}, \hat{p}_{0}, p^{0}, w\right)=0$. Consider the set

$$
X=\left\{x_{0} \in O \mid \max _{|w| \leqslant 1} H\left(x_{0}, \alpha^{\prime}\left(x_{0}\right), p^{0}, w\right)=0\right\} .
$$

We claim that $X$ is a $(n-1)$-dimensional manifold ${ }^{6}$. Indeed, consider the map

$$
\begin{aligned}
G: O & \rightarrow \mathbb{R} \\
x_{0} & \mapsto G\left(x_{0}\right)=\max _{|w| \leqslant 1} H\left(x_{0}, \alpha^{\prime}\left(x_{0}\right), p^{0}, w\right)
\end{aligned}
$$

and the vector field $h_{1}\left(x_{0}\right)=X\left(x_{0}\right)+u_{1} Y_{1}\left(x_{0}\right)$ that defines the extremal trajectory $x(\cdot)$ on the interval $\left[0, \tau_{1}\left(x_{0}\right)\right)$, associated to the value $u_{1}$ that satisfies the maximization condition (4) on the referred interval. Proving that $X$ is a $(n-1)$-dimensional manifold amounts to proving that, for every function $\alpha \in C^{2}$ such that $\alpha^{\prime}\left(x_{0}\right)=p_{0}$, there holds $d G\left(x_{0}\right) \neq 0$ before the first conjugate time $t_{c}$. The second variation formula given in [38, p. 275, equation (12)] taken at $(\delta x, \varepsilon)=\left(h_{1}\left(x_{0}\right),-1,0, \ldots, 0\right)$ is equal to, after some simplifications, $d G\left(x_{0}\right) \cdot h_{1}\left(x_{0}\right)$. Since the second variation is positive definite on $\left(0, t_{c}\right)$ then $d G\left(x_{0}\right) \cdot h_{1}\left(x_{0}\right) \neq 0$ before $t_{c}$. The claim is proved.

Define the $j^{\text {th }}$ switching surface $\Sigma_{j}$, for $j=1, \ldots, s$, as the image of the mapping

$$
x_{0} \mapsto \exp \left(\tau_{j}\left(x_{0}\right), x_{0}, \alpha^{\prime}\left(x_{0}\right)\right),
$$

where $x_{0} \in X$.

Remark 1.3. If the strict bang-bang Legendre condition holds, then the flow associated to the maximized Hamiltonian crosses the switching surface $\Sigma_{j}$ at the instant $\hat{\tau}_{j}$ transversally, for $j=1, \ldots, s$ (see [3]).

Theorem $2([3,29,30,33])$. Let $\left(\hat{x}(\cdot), \hat{p}(\cdot), p^{0}, \hat{u}_{1}(\cdot)\right)$ be a bang-bang extremal for $(\boldsymbol{O C P})$ that satisfies the strict bang-bang Legendre condition on $\left[0, t_{c}\right)$, with $t_{c}<+\infty$. The trajectory $\hat{x}(\cdot)$ is strong locally optimal if and only if there exists a function $\alpha \in C^{2}$ with $\alpha^{\prime}\left(\hat{x}_{0}\right)=\hat{p}_{0}$ such that:

- the trajectory $\hat{x}(\cdot)$ can be embedded into the field of non-intersecting (broken) extremals $\left(t, x_{0}\right) \mapsto$ $\exp \left(t, x_{0}, \alpha^{\prime}\left(x_{0}\right)\right)$ where $x_{0} \in O$;

\footnotetext{
${ }^{6}$ The argument that follows is due to L. Poggiolini.
}

- this field of extremals crosses the switching surfaces $\Sigma_{j}$ transversally, for $j=1, \ldots, s$, and for $j=1, \ldots, s+1$, with $\tau_{s+1}\left(\hat{x}_{0}\right)=t_{c}$, the mapping

$$
\begin{aligned}
\left(\tau_{j-1}\left(x_{0}\right), \tau_{j}\left(x_{0}\right)\right) \times X & \longrightarrow \mathbb{R}^{n} \\
\left(t, x_{0}\right) & \longmapsto \exp \left(t, x_{0}, \alpha^{\prime}\left(x_{0}\right)\right)
\end{aligned}
$$

is of rank $n$.

Remark 1.4. In the conditions of Theorem 2, at the first conjugate point $\hat{x}\left(t_{c}\right)$, the flow of the extremal field reflects off the switching surface, causing an overlap of the flow near this surface (see Figure 1 - switching surface $\Sigma_{s+1}$, and see [20,33]).

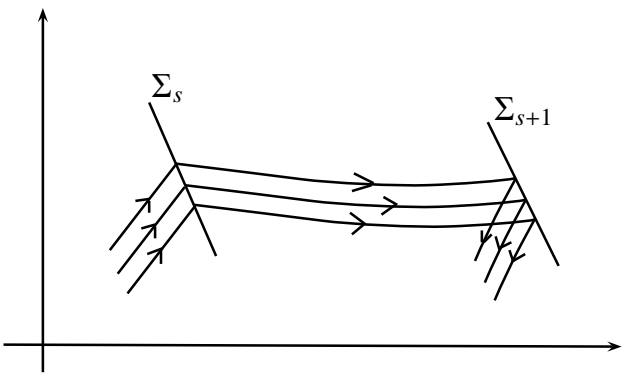

Figure 1: Field of extremals

Remark 1.5. Let $f_{j}\left(x_{0}\right)=X\left(x_{0}\right)+u_{j} Y_{1}\left(x_{0}\right)$, for $j=$ $1, \ldots, s+2$ and $x_{0} \in O$, be the vector fields that define the extremal trajectory $x(\cdot)$ on $\left(\tau_{j-1}\left(x_{0}\right), \tau_{j}\left(x_{0}\right)\right)$, with $\tau_{s+1}\left(\hat{x}_{0}\right)=t_{c}$ and where $u_{j}$ is the value $(1$ or -1$)$ of the control that satisfies the maximization condition (4) in each respective interval. If we take $x_{0} \in X$ and $j=1, \ldots, s+1$, then for $\left(t, x_{0}\right) \in\left(\tau_{j-1}, \tau_{j}\right) \times X$

$$
\operatorname{det}\left(\exp \left(t, x_{0}, \alpha^{\prime}\left(x_{0}\right)\right), f_{j}\left(x_{0}\right)\right)
$$

has constant sign (see [38]).

Moreover, the determinants

$\operatorname{det}\left(\left.\frac{d}{d x_{0}} \exp \left(t, x_{0}, \alpha^{\prime}\left(x_{0}\right)\right)\right|_{\left(t, x_{0}\right) \in\left(\tau_{s}\left(x_{0}\right), \tau_{s+1}\left(x_{0}\right)\right) \times X}, f_{s+1}\left(x_{0}\right)\right)$

and

$\operatorname{det}\left(\left.\frac{d}{d x_{0}} \exp \left(t, x_{0}, \alpha^{\prime}\left(x_{0}\right)\right)\right|_{\left(t, x_{0}\right) \in\left(\tau_{s+1}\left(x_{0}\right), \tau_{s+2}\left(x_{0}\right)\right) \times X}, f_{s+2}\left(x_{0}\right)\right)$

have different signs (see [38]).

The computation of conjugate times in the bang-bang case is difficult in practice. In the last years several 
methods have been developed for the numerical implementation of second order sufficient optimality conditions (see, e.g., [27, 30] and references cited therein). These numerical procedures allow the computation of the first conjugate time, for bang-bang optimal control problems with affine-control systems, whenever it exists and is attained at a $j^{\text {th }}$ switching time. Besides, in the smooth case, efficient tools are available, see e.g. [11]. We next propose a regularization procedure which allows the use of these tools for the computation of the first conjugate time for the problem (OCP). However, in practice, if $j$ is too large then the numerical computation of the first conjugate time may become very difficult either using the methods for bang-bang or smooth controls.

\subsection{The regularization procedure}

Let $\varepsilon$ be a positive real parameter and let $Y_{2}, \ldots, Y_{m}$ be $m-1$ arbitrary smooth vector fields on $\mathbb{R}^{n}$, where $m \geqslant 2$ is an integer. Consider the control-affine system

$$
\dot{x}^{\varepsilon}(t)=X\left(x^{\varepsilon}(t)\right)+u_{1}^{\varepsilon}(t) Y_{1}\left(x^{\varepsilon}(t)\right)+\varepsilon \sum_{i=2}^{m} u_{i}^{\varepsilon}(t) Y_{i}\left(x^{\varepsilon}(t)\right),
$$

where the control $u^{\varepsilon}(t)=\left(u_{1}^{\varepsilon}(t), \ldots, u_{m}^{\varepsilon}(t)\right)$ satisfies the constraint

$$
\sum_{i=1}^{m}\left(u_{i}^{\varepsilon}(t)\right)^{2} \leqslant 1
$$

Consider the optimal control problem $(\mathbf{O C P})_{\varepsilon}$ of determining a trajectory $x^{\varepsilon}(\cdot)$, solution of (7)-(8) on $\left[0, t_{f}^{\varepsilon}\right]$, such that $x^{\varepsilon}(0)=\hat{x}_{0}$ and $x^{\varepsilon}\left(t_{f}^{\varepsilon}\right)=\hat{x}_{1}$, and minimizing the time of transfer $t_{f}^{\varepsilon}$. The parameter $\varepsilon$ is viewed as a penalization parameter. The existence of at least one solution for $(\mathbf{O C P})_{\varepsilon}$ is proved in [44], and it is also proved that any solution $x^{\varepsilon}(\cdot)$ of $(\mathbf{O C P})_{\varepsilon}$ converges uniformly on $\left[0, t_{f}\right]$ to a solution $x(\cdot)$ of $(\mathbf{O C P})$ when $\varepsilon$ tends to zero (see Theorem 4).

According to the Pontryagin maximum principle, any optimal solution $\hat{x}^{\varepsilon}(\cdot)$ of $(\mathbf{O C P})_{\varepsilon}$, associated with controls $\left(\hat{u}_{1}^{\varepsilon}, \ldots, \hat{u}_{m}^{\varepsilon}\right)$ satisfying the constraint (8), is the projection of an extremal $\left(\hat{x}^{\varepsilon}(\cdot), \hat{p}^{\varepsilon}(\cdot), p^{0 \varepsilon}, \hat{u}^{\varepsilon}(\cdot)\right)$ such that

$$
\begin{aligned}
\dot{\hat{p}}^{\varepsilon}(t)= & -\frac{\partial H^{\varepsilon}}{\partial x}\left(\hat{x}^{\varepsilon}(t), \hat{p}^{\varepsilon}(t), p^{0 \varepsilon}, \hat{u}^{\varepsilon}(t)\right) \\
= & -\left\langle\hat{p}^{\varepsilon}(t), \frac{\partial X}{\partial x}\left(\hat{x}^{\varepsilon}(t)\right)\right\rangle-\hat{u}_{1}^{\varepsilon}(t)\left\langle\hat{p}^{\varepsilon}(t), \frac{\partial Y_{1}}{\partial x}\left(\hat{x}^{\varepsilon}(t)\right)\right\rangle \\
& -\varepsilon \sum_{i=2}^{m} \hat{u}_{i}^{\varepsilon}(t)\left\langle\hat{p}^{\varepsilon}(t), \frac{\partial Y_{i}}{\partial x}\left(\hat{x}^{\varepsilon}(t)\right)\right\rangle
\end{aligned}
$$

where $H^{\varepsilon}\left(x^{\varepsilon}, p^{\varepsilon}, p^{0 \varepsilon}, u^{\varepsilon}\right)=\left\langle p^{\varepsilon}, X\left(x^{\varepsilon}\right)+u_{1}^{\varepsilon} Y_{1}\left(x^{\varepsilon}\right)+\right.$ $\left.\varepsilon \sum_{i=2}^{m} u_{i}^{\varepsilon} Y_{i}\left(x^{\varepsilon}\right)\right\rangle+p^{0 \varepsilon}$ is the Hamiltonian, and

$H^{\varepsilon}\left(\hat{x}^{\varepsilon}(t), \hat{p}^{\varepsilon}(t), p^{0 \varepsilon}, \hat{u}^{\varepsilon}(t)\right)=\max _{\sum_{i=1}^{m} w_{i}^{2} \leqslant 1} H^{\varepsilon}\left(\hat{x}^{\varepsilon}(t), \hat{p}^{\varepsilon}(t), p^{0 \varepsilon}, w\right)$

almost everywhere on $\left[0, t_{f}^{\varepsilon}\right]$. Moreover, the maximized Hamiltonian is equal to 0 on $\left[0, t_{f}^{\varepsilon}\right]$. The maximization condition (10) turns into

$$
\begin{aligned}
& \hat{u}_{1}^{\varepsilon}(t)\left\langle\hat{p}^{\varepsilon}(t), Y_{1}\left(\hat{x}^{\varepsilon}(t)\right)\right\rangle+\varepsilon \sum_{i=2}^{m} \hat{u}_{i}^{\varepsilon}(t)\left\langle\hat{p}^{\varepsilon}(t), Y_{i}\left(\hat{x}^{\varepsilon}(t)\right)\right\rangle \\
& =\max _{\sum_{i=1}^{m} w_{i}^{2} \leqslant 1}\left(w_{1}\left\langle\hat{p}^{\varepsilon}(t), Y_{1}\left(\hat{x}^{\varepsilon}(t)\right)\right\rangle+\varepsilon \sum_{i=2}^{m} w_{i}\left\langle\hat{p}^{\varepsilon}(t), Y_{i}\left(\hat{x}^{\varepsilon}(t)\right)\right\rangle\right),
\end{aligned}
$$

and two cases may occur: either the maximum is attained in the interior of the domain, or it is attained at the boundary. In the first case, there must hold $\left\langle p^{\varepsilon}(t), Y_{i}\left(x^{\varepsilon}(t)\right)\right\rangle=0$, for every $i \in\{1, \ldots, m\}$; in particular, if the $m$ functions $t \mapsto\left\langle p^{\varepsilon}(t), Y_{i}\left(x^{\varepsilon}(t)\right)\right\rangle, i=1, \ldots, m$, do not vanish simultaneously, then the maximum is attained on the boundary of the domain.

We make the following assumption.

Assumption 3. The integer $m$ and the vector fields $Y_{2}, \ldots, Y_{m}$ are chosen such that

$$
\operatorname{Span}\left\{Y_{i} \mid i=1, \ldots, m\right\}=\mathbb{R}^{n} .
$$

Under the Assumption 3, the maximization condition (11) yields, for $i=2, \ldots, m$,

$$
\begin{aligned}
& \hat{u}_{1}^{\varepsilon}(t)=\frac{\left\langle\hat{p}^{\varepsilon}(t), Y_{1}\left(\hat{x}^{\varepsilon}(t)\right)\right\rangle}{\sqrt{\left\langle\hat{p}^{\varepsilon}(t), Y_{1}\left(\hat{x}^{\varepsilon}(t)\right)\right\rangle^{2}+\varepsilon^{2} \sum_{i=2}^{m}\left\langle\hat{p}^{\varepsilon}(t), Y_{i}\left(\hat{x}^{\varepsilon}(t)\right)\right\rangle^{2}}}, \\
& \hat{u}_{i}^{\varepsilon}(t)=\frac{\varepsilon\left\langle\hat{p}^{\varepsilon}(t), Y_{i}\left(\hat{x}^{\varepsilon}(t)\right)\right\rangle}{\sqrt{\left\langle\hat{p}^{\varepsilon}(t), Y_{1}\left(\hat{x}^{\varepsilon}(t)\right)\right\rangle^{2}+\varepsilon^{2} \sum_{i=2}^{m}\left\langle\hat{p}^{\varepsilon}(t), Y_{i}\left(\hat{x}^{\varepsilon}(t)\right)\right\rangle^{2}}},
\end{aligned}
$$

for almost every $t \in\left[0, t_{f}^{\varepsilon}\right]$, and moreover the control functions $\hat{u}_{i}^{\varepsilon}(\cdot), i=1, \ldots, m$ are smooth functions of $t$ (so that the above formula holds actually for every $t \in$ $\left.\left[0, t_{f}^{\varepsilon}\right]\right)$.

Note that this assumption implies that $m \geqslant n$. Actually, this assumption can be weakened (see [44] for details).

Theorem 4 ([44]). Assume that the problem (OCP) has a unique solution $\hat{x}(\cdot)$, defined on $\left[0, t_{f}\right]$, associated 
with a bang-bang control $\hat{u}_{1}(\cdot)$ on $\left[0, t_{f}\right]$. Moreover, assume that $\hat{x}(\cdot)$ has a unique extremal lift (up to a multiplicative scalar), which is moreover normal, denoted $\left(\hat{x}(\cdot), \hat{p}(\cdot),-1, \hat{u}_{1}(\cdot)\right)$.

Then, under the Assumption 3, there exists $\varepsilon_{0}>0$ such that, for every $\varepsilon \in\left(0, \varepsilon_{0}\right)$, the problem $(\boldsymbol{O C P})_{\varepsilon}$ has at least one solution $\hat{x}^{\varepsilon}(\cdot)$, defined on $\left[0, t_{f}^{\varepsilon}\right]$ with $t_{f}^{\varepsilon} \leqslant t_{f}$, associated with a smooth control $u^{\varepsilon}(\cdot)=$ $\left(u_{1}^{\varepsilon}(\cdot), \ldots, u_{m}^{\varepsilon}(\cdot)\right)$ satisfying the constraint $(8)$, every extremal lift of which is normal. Let $\left(\hat{x}^{\varepsilon}(\cdot), \hat{p}^{\varepsilon}(\cdot),-1, \hat{u}^{\varepsilon}(\cdot)\right)$ be such a normal extremal lift. Then, as $\varepsilon$ tends to 0 ,

- $t_{f}^{\varepsilon}$ converges to $t_{f}$;

- $\hat{x}^{\varepsilon}(\cdot)$ converges uniformly $y^{7}$ to $\hat{x}(\cdot)$, and $\hat{p}^{\varepsilon}(\cdot)$ converges uniformly to $\hat{p}(\cdot)$ on $\left[0, t_{f}\right]$;

- $\hat{u}_{1}^{\varepsilon}(\cdot)$ converges to $\hat{u}_{1}(\cdot)$ and $\hat{u}_{i}^{\varepsilon}(\cdot), i=2, \ldots, m$, converge to 0 almost everywhere on $\left[0, t_{f}\right]$, and thus in particular for the strong $L^{1}\left(0, t_{f}\right)$ topology.

Remark 1.6. This result remains true if we extend forward the interval $\left[0, t_{f}\right]$ on an interval $[0, T]$ for $T \in I$, where $I$ is a maximal time interval of $[0,+\infty)$ containing $\left[0, t_{f}\right]$.

Remark 1.7. It is assumed that the problem (OCP) has a unique solution $\hat{x}(\cdot)$, having a unique extremal lift that is normal. Such an assumption holds true whenever the minimum time function (the value function of the optimal control problem) enjoys differentiability properties (see e.g. [7, 15] for a precise relationship, see also $[13,40,41,45]$ for results on the size of the set where the value function is differentiable).

\subsection{Conjugate times in the smooth case}

We recall how to define the concept of first conjugate time for the smooth optimal control problem $(\mathbf{O C P})_{\varepsilon}$. A first possible definition of conjugate times is in terms of a quadratic form, which is the second order intrinsic derivative of the end-point mapping defined by $E\left(\varepsilon, t_{f}^{\varepsilon}, \hat{x}_{0}, u^{\varepsilon}\right)=x^{\varepsilon}\left(t_{f}^{\varepsilon}\right)$ where $t \mapsto x^{\varepsilon}\left(\varepsilon, t, \hat{x}_{0}, u^{\varepsilon}\right)$ is the trajectory solution of (7), associated to the control $u^{\varepsilon}$, such that $x^{\varepsilon}\left(\varepsilon, 0, \hat{x}_{0}, u^{\varepsilon}\right)=\hat{x}_{0}$. Testing a conjugate time amounts to testing the positivity of that quadratic form. However, this definition requires a corank one assumption, and we will rather use a geometric concept of conjugate time, defined below. We refer the reader to [11] for a survey on that theory and to [5] for extensive explanations and for the more general Morse index theory.

\footnotetext{
${ }^{7} \mathrm{We}$ consider any continuous extension of the extremal $\left(\hat{x}^{\varepsilon}(\cdot), \hat{p}^{\varepsilon}(\cdot),-1, \hat{u}_{1}^{\varepsilon}(\cdot)\right)$ on $\left[0, t_{f}\right]$.
}

Geometric conjugate time.

Definition 1.4. Let $x_{0} \in O$. The point $x^{\varepsilon}\left(t_{c}^{\varepsilon}\right)$ is geometrically conjugate to $x^{\varepsilon}(0)$ if and only if the mapping $x_{0} \mapsto \exp ^{\varepsilon}\left(t_{c}^{\varepsilon}, x_{0}, \alpha^{\prime}\left(x_{0}\right)\right)$ is not immersive, that is,

$$
\operatorname{det}\left(\frac{d}{d x_{0}} \exp ^{\varepsilon}\left(t_{c}^{\varepsilon}, x_{0}, \alpha^{\prime}\left(x_{0}\right)\right)\right)=0 .
$$

The time $t_{c}^{\varepsilon}$ is called a geometric conjugate time.

Remark 1.8. Given an extremal $\left(\hat{x}^{\varepsilon}(\cdot), \hat{p}^{\varepsilon}(\cdot), p^{0 \varepsilon}, u^{\varepsilon}(\cdot)\right)$, the notion of geometric conjugate time coincides with the notion of conjugate time defined in terms of quadratic form, provided the following assumptions hold:

- the strong Legendre condition holds along the extremal, that is, there exists $\gamma>0$ such that

$$
\frac{\partial^{2} H}{\partial u^{2}}\left(\hat{x}^{\varepsilon}(\cdot), \hat{p}^{\varepsilon}(\cdot), p^{0 \varepsilon}, u_{1}^{\varepsilon}(\cdot)\right) \cdot(v, v) \leqslant-\gamma\|v\|^{2},
$$

for every $v \in \mathbb{R}^{m}$;

- the control $u^{\varepsilon}$ is of corank one on every subinterval (assumption of strong regularity, see [42]).

Moreover, in that case the first conjugate time $t_{c}^{\varepsilon}$ characterizes the optimality status of the extremal: the trajectory $\hat{x}^{\varepsilon}(\cdot)$ is strongly locally optimal on $[0, t]$, for every $t<t_{c}^{\varepsilon}$; for $t>t_{c}^{\varepsilon}$, the trajectory $\hat{x}^{\varepsilon}(\cdot)$ is not locally optimal on $[0, t]$ (see, e.g., $[5,11,42])$.

Remark 1.9. None of the two assumptions of the previous remark will be made for the extremal $\left(\hat{x}^{\varepsilon}(\cdot), \hat{p}^{\varepsilon}(\cdot), p^{0 \varepsilon}, \hat{u}^{\varepsilon}(\cdot)\right)$. In fact, our aim is to prove that the first geometric conjugate time $t_{c}^{\varepsilon}$ converges to the first conjugate time $t_{c}$ of the bang-bang case, when $\varepsilon$ tends to 0 . This result, derived in Theorem 6 (Section 1.5), will permit to use as well in the bang-bang case the available efficient implementation procedures that exist in the smooth case, like for instance the free package $\operatorname{COTCOT}^{8}$ (see [11]).

For normal extremals $\left(x^{\varepsilon}(\cdot), p^{\varepsilon}(\cdot),-1, u^{\varepsilon}(\cdot)\right)$ that satisfy the strong Legendre condition, the absence of conjugate points is a sufficient condition for local optimality (see e.g. [5]). This sufficient optimality condition will be expressed using the extremal field approach.

\footnotetext{
${ }^{8}$ Conditions of Order Two, COnjugate Times, http://apo.enseeiht.fr/cotcot/
} 
Extremal field approach. From Theorem 4 every extremal lift of the problem $(\mathbf{O C P})_{\varepsilon}$ is normal $\left(p^{0 \varepsilon}=\right.$ -1 ). Analogously to the bang-bang case, the aim is to construct a family of extremals containing the reference normal extremal $\left(\hat{x}^{\varepsilon}(\cdot), \hat{p}^{\varepsilon}(\cdot),-1, \hat{u}^{\varepsilon}(\cdot)\right)$, sharing nice non-intersection properties before the first conjugate time.

For every $z_{0}=\left(x_{0}, p_{0}\right) \in \mathbb{R}^{n} \times \mathbb{R}^{n}$, let $z^{\varepsilon}\left(\cdot, z_{0}\right)=$ $\left(x^{\varepsilon}\left(\cdot, z_{0}\right), p^{\varepsilon}\left(\cdot, z_{0}\right)\right)$ be the solution of the system of equations (7) and (9), with the controls (12), such that $z^{\varepsilon}\left(0, z_{0}\right)=z_{0}$. The exponential mapping associated to $(\mathbf{O C P})_{\varepsilon}$ is defined by

$$
\exp ^{\varepsilon}\left(t, z_{0}\right)=x^{\varepsilon}\left(t, z_{0}\right) .
$$

Let $x_{0} \in O$ and $\alpha^{\varepsilon}: \mathbb{R}^{n} \rightarrow \mathbb{R}$ be a $C^{2}$ function such that $\alpha^{\varepsilon^{\prime}}\left(x_{0}\right)=p^{\varepsilon}(0)$, and such that the family of functions $\left(\alpha^{\varepsilon}\right)$ converges to the function $\alpha$ associated with the problem (OCP) in $C^{2}$ topology, as $\varepsilon$ tends to 0 . As in the bang-bang case, define

$$
X^{\varepsilon}=\left\{x_{0} \in O \mid \max _{\sum_{i=1}^{m} w_{i}^{2} \leqslant 1} H^{\varepsilon}\left(x_{0}, \alpha^{\varepsilon^{\prime}}\left(x_{0}\right),-1, w^{\varepsilon}\right)=0\right\} .
$$

For $\varepsilon>0$ small enough, $X^{\varepsilon}$ is a $(n-1)$-dimensional manifold. Indeed, let $G^{\varepsilon}$ be defined on $O$ by $G^{\varepsilon}\left(x_{0}\right)=$ $\max _{\sum_{i=1}^{m} w_{i}^{2} \leqslant 1} H^{\varepsilon}\left(x_{0}, \alpha^{\prime}\left(x_{0}\right),-1, w^{\varepsilon}\right)$. It follows from Theorem 4 that $G^{\varepsilon}$ converges to $G$ (defined in Section 1.2) as $\varepsilon$ goes to 0 , and therefore, for $\alpha \in C^{2}$ such that $\alpha^{\prime}\left(x_{0}\right)=p_{0}$, there holds $d G^{\varepsilon}\left(x_{0}\right) \neq 0$, since $d G\left(x_{0}\right) \neq 0$.

Theorem 5 ([5]). If the normal extremal $\left(\hat{x}^{\varepsilon}(\cdot), \hat{p}^{\varepsilon}(\cdot),-1, \hat{u}^{\varepsilon}(\cdot)\right)$ satisfies the strong Legendre condition and, moreover can be embedded into the family of extremals $\exp ^{\varepsilon}\left(t, x_{0}, \alpha^{\varepsilon^{\prime}}\left(x_{0}\right)\right)$ such that the mapping

$$
\begin{aligned}
\left(0, t_{c}^{\varepsilon}\right) \times X^{\varepsilon} & \rightarrow \mathbb{R}^{n} \\
\left(t, x_{0}\right) & \mapsto \exp ^{\varepsilon}\left(t, x_{0}, \alpha^{\varepsilon^{\prime}}\left(x_{0}\right)\right)
\end{aligned}
$$

is of rank $n$, then $\left(\hat{x}^{\varepsilon}(\cdot), \hat{p}^{\varepsilon}(\cdot),-1, \hat{u}^{\varepsilon}(\cdot)\right)$ is a local minimum in $C^{0}$ topology for the problem $(\boldsymbol{O C P})_{\varepsilon}$.

Remark 1.10. The typical behavior of the flow of the extremal field at the first conjugate point is a fold point (see Figure 2, and see [5, 18]).

Remark 1.11. If one considers $x_{0} \in X^{\varepsilon}$, then $x^{\varepsilon}\left(t_{c}^{\varepsilon}\right)$ is geometrically conjugate to $x^{\varepsilon}(0)$ if and only if

$$
\operatorname{det}\left(\frac{d}{d x_{0}} \exp ^{\varepsilon}\left(t_{c}^{\varepsilon}, x_{0}, \alpha^{\varepsilon^{\prime}}\left(x_{0}\right)\right)_{\mid X^{\varepsilon}}, f^{\varepsilon}\left(x_{0}\right)\right)=0,
$$

where $f^{\varepsilon}\left(x_{0}\right)=X\left(x^{\varepsilon}\left(x_{0}\right)\right)+\varepsilon \sum_{i=1}^{m} u_{i}^{\varepsilon}\left(x_{0}, \alpha^{\varepsilon^{\prime}}\left(x_{0}\right)\right)$ and $u_{i}^{\varepsilon}\left(x_{0}, \alpha^{\varepsilon^{\prime}}\left(x_{0}\right)\right)$ are smooth functions that satisfy the maximization condition (11).

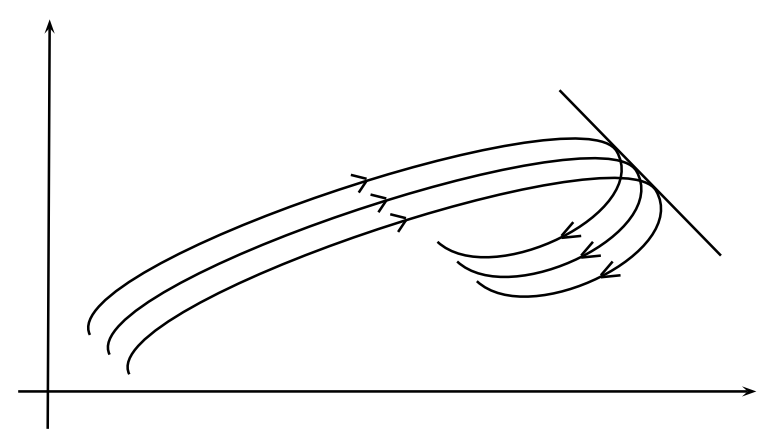

Figure 2: Field of extremals in the smooth case

Remark 1.12. Note that, as long as the minimum time function is differentiable at the point $\hat{x}^{\varepsilon}(t)$, the optimal trajectory $\hat{x}^{\varepsilon}(\cdot)$ can be embedded into a non-intersecting extremal field.

Remark 1.13. To derive a necessary optimality condition, a corank one assumption is required for the extremal $\left(\hat{x}^{\varepsilon}(\cdot), \hat{p}^{\varepsilon}(\cdot), p^{0 \varepsilon}, \hat{u}^{\varepsilon}(\cdot)\right)$ (see [11]).

\subsection{Main result}

We first recall the context. Let $\hat{x}(\cdot)$ denote the strong locally optimal trajectory of (OCP), corresponding to the control $\hat{u}_{1}$ on $\left[0, t_{f}\right]$. In particular, $t_{f}$ is the minimal time so that $\hat{x}(0)=\hat{x}_{0}$ and $\hat{x}\left(t_{f}\right)=\hat{x}_{1}$. We extend $\hat{x}(\cdot)$ on a maximal interval $I \subset[0,+\infty)$ containing $\left[0, t_{f}\right]$, and denote by $t_{c}$ its first conjugate time. For every $\varepsilon>0$, let $\hat{x}^{\varepsilon}(\cdot)$ denote an optimal trajectory solution of $(\mathbf{O C P})_{\varepsilon}$, corresponding to a control $\hat{u}^{\varepsilon}=\left(\hat{u}_{1}^{\varepsilon}, \ldots, \hat{u}_{m}^{\varepsilon}\right)$ on $\left[0, t_{f}^{\varepsilon}\right]$. In particular, $t_{f}^{\varepsilon}$ is the minimal time so that $\hat{x}^{\varepsilon}(0)=\hat{x}_{0}$ and $\hat{x}^{\varepsilon}\left(t_{f}^{\varepsilon}\right)=\hat{x}_{1}$. We extend $\hat{x}^{\varepsilon}(\cdot)$ on a maximal interval of $[0,+\infty)$ containing $\left[0, t_{f}^{\varepsilon}\right]$, and denote by $t_{c}^{\varepsilon}$ its first geometrically conjugate time.

Theorem 6. Assume that the problem (OCP) has a unique solution $\hat{x}(\cdot)$, associated with a bang-bang control $\hat{u}_{1}(\cdot)$, on a maximal interval I. Moreover, assume that $\hat{x}(\cdot)$ has a unique extremal lift (up to a multiplicative scalar), which is moreover normal, and denoted by $\left(\hat{x}(\cdot), \hat{p}(\cdot),-1, \hat{u}_{1}(\cdot)\right)$. If the extremal $\left(\hat{x}(\cdot), \hat{p}(\cdot),-1, \hat{u}_{1}(\cdot)\right)$ satisfies, moreover, the strict bang-bang Legendre condition on $\left[0, t_{c}\right]$, then the first geometric conjugate time $t_{c}^{\varepsilon}$ converges to the first conjugate time $t_{c}$ as $\varepsilon$ tends to 0 .

Remark 1.14. Let $t_{\text {cut }}$ denote the cut time along the extremal $\left(\hat{x}(\cdot), \hat{p}(\cdot), p^{0}, \hat{u}(\cdot)\right)$. Analogously to the bangbang case, we can define the cut time $t_{\text {cut }}^{\varepsilon}$ of the optimal trajectory $\hat{x}^{\varepsilon}(\cdot)$ for the problem $(\mathbf{O C P})_{\varepsilon}$ as the first time at which $\hat{x}^{\varepsilon}(\cdot)$ loses its optimality. We claim 
that, under the assumptions of Theorem 4, there holds $\limsup _{\varepsilon \rightarrow 0} t_{\text {cut }}^{\varepsilon} \leqslant t_{\text {cut }}$.

\section{Proof of the main result}

The next proposition is the key result to derive Theorem 6.

Proposition 7. Let $O$ be a neighborhood of $\hat{x}_{0}$ and $x_{0} \in O$. The exponential mapping $\left(t, x_{0}\right) \mapsto \exp ^{\varepsilon}\left(t, x_{0}, \alpha^{\varepsilon^{\prime}}\left(x_{0}\right)\right)$ converges to $\left(t, x_{0}\right) \mapsto \exp \left(t, x_{0}, \alpha^{\prime}\left(x_{0}\right)\right)$ piecewise in $C^{1}$ topology on $I \times O$, with $\tau_{s+1}\left(\hat{x}_{0}\right)=t_{c}$, as $\varepsilon$ tends to 0 . More precisely, on every compact subinterval of $\left(\tau_{j-1}\left(x_{0}\right), \tau_{j}\left(x_{0}\right)\right) \times O$, with $\left(\tau_{j-1}\left(x_{0}\right), \tau_{j}\left(x_{0}\right)\right) \subset I$ and $j \in \mathbb{N}$, the mapping $\left(t, x_{0}\right) \mapsto \exp ^{\varepsilon}\left(t, x_{0}, \alpha^{\varepsilon^{\prime}}\left(x_{0}\right)\right)$ converges to $\left(t, x_{0}\right) \mapsto \exp \left(t, x_{0}, \alpha^{\prime}\left(x_{0}\right)\right)$ uniformly in $C^{1}$ topology.

Proof. In what follows, when it is convenient, we simplify the notation and write $\exp \left(t, x_{0}\right)$ or $x\left(t, x_{0}\right)$ (respectively, $\exp ^{\varepsilon}\left(t, x_{0}\right)$ or $\left.x^{\varepsilon}\left(t, x_{0}\right)\right)$ for $\exp \left(t, x_{0}, \alpha^{\prime}\left(x_{0}\right)\right)$ (respectively, for $\left.\exp ^{\varepsilon}\left(t, x_{0}, \alpha^{\varepsilon \prime}\left(x_{0}\right)\right)\right)$.

Let $\varepsilon>0$ small enough. For $x_{0} \in O$, consider the function

$$
\varphi_{1}\left(\varepsilon, t, x_{0}\right)=\left\langle p\left(\varepsilon, t, x_{0}\right), Y_{1}\left(x\left(\varepsilon, t, x_{0}\right)\right)\right\rangle .
$$

For $\left(\varepsilon, t, x_{0}\right)=\left(0, \hat{\tau}_{j}, x_{0}\right)$, by definition of the switching time, one has $\varphi_{1}\left(0, \hat{\tau}_{j}, x_{0}\right)=0$, and by the strict bang-bang Legendre condition, $\frac{\partial \varphi_{1}}{\partial t}\left(0, \hat{\tau}_{j}, x_{0}\right) \neq 0$. By the implicit function theorem there exists a neighborhood $\left(-\varepsilon_{0}, \varepsilon_{0}\right)$ of $0 \in \mathbb{R}$, such that for $\varepsilon \in\left(-\varepsilon_{0}, \varepsilon_{0}\right)$, there exists a $C^{1}$ function $\tau_{j}^{\varepsilon}\left(x_{0}\right)=\tau_{j}^{\varepsilon}\left(\varepsilon, x_{0}\right)$, with $j=$ $1, \ldots, s$, satisfying $\varphi_{1}\left(\varepsilon, \tau_{j}^{\varepsilon}\left(x_{0}\right)\right)=0$ and such that, as $\varepsilon$ tends to $0, \tau_{j}^{\varepsilon}\left(x_{0}\right)$ converges to $\tau_{j}\left(x_{0}\right)$, and $\frac{\partial \tau_{j}^{\varepsilon}}{\partial x_{0}}\left(x_{0}\right)$ converges to $\frac{\partial \tau_{j}}{\partial x_{0}}\left(x_{0}\right)$.

Analogously to the definition of switching time function of an extremal trajectory $x(\cdot)$, we have thus defined some functions $\tau_{j}^{\varepsilon}(\cdot): O \rightarrow \mathbb{R}$, that are however not switching functions.

Lemma 2.1. The mapping $\left(t, x_{0}\right) \mapsto \exp ^{\varepsilon}\left(t, x_{0}, \alpha^{\varepsilon^{\prime}}\left(x_{0}\right)\right)$ converges to $\left(t, x_{0}\right) \mapsto \exp \left(t, x_{0}, \alpha^{\prime}\left(x_{0}\right)\right)$ uniformly in $C^{1}$ topology on $J \times O$, where $J$ is any compact subinterval of $\left[0, \tau_{1}\left(x_{0}\right)\right)$, as $\varepsilon$ tends to 0 .

Proof. Let $J$ be a compact subinterval of $\left[0, \tau_{1}\left(x_{0}\right)\right)$. The uniform $C^{0}$ convergence on $J \times O$ of the mapping $\left(t, x_{0}\right) \mapsto \exp ^{\varepsilon}\left(t, x_{0}\right)$ to $\left(t, x_{0}\right) \mapsto \exp \left(t, x_{0}\right)$, as $\varepsilon$ tends to 0 , is a direct consequence of Theorem 4 . We have

$$
\frac{\partial \exp ^{\varepsilon}}{\partial t}\left(t, x_{0}\right)=\dot{x}^{\varepsilon}\left(t, x_{0}\right)
$$

where $\dot{x}^{\varepsilon}\left(t, x_{0}\right)$ is given by (7); from Theorem $4, \dot{x}^{\varepsilon}\left(t, x_{0}\right)$ converges to $\dot{x}\left(t, x_{0}\right)=\frac{d \exp }{d t}\left(t, x_{0}\right)$ as $\varepsilon$ tend to 0 . On the other hand,

$$
\begin{array}{r}
\frac{d}{d x_{0}} \exp ^{\varepsilon}\left(t, x_{0}, \alpha^{\varepsilon^{\prime}}\left(x_{0}\right)\right)=\frac{\partial \exp ^{\varepsilon}}{\partial x_{0}}\left(t, x_{0}, \alpha^{\varepsilon^{\prime}}\left(x_{0}\right)\right) \\
+\frac{\partial \exp ^{\varepsilon}}{\partial p_{0}}\left(t, x_{0}, \alpha^{\varepsilon^{\prime}}\left(x_{0}\right)\right) \alpha^{\varepsilon^{\prime \prime}}\left(x_{0}\right)
\end{array}
$$

where $\frac{\partial \exp ^{\varepsilon}}{\partial x_{0}}\left(t, x_{0}, \alpha^{\varepsilon^{\prime}}\left(x_{0}\right)\right)$ and $\frac{\partial \exp ^{\varepsilon}}{\partial p_{0}}\left(t, x_{0}, \alpha^{\varepsilon^{\prime}}\left(x_{0}\right)\right)$ are solutions of the linearized system associated with the Hamiltonian system (7)-(9), for the problem $(\mathbf{O C P})_{\varepsilon}$ on $[0, t]$. From Theorem 4, $\left(x^{\varepsilon}(\cdot), p^{\varepsilon}(\cdot)\right)$ converges uniformly to the solution of the Hamiltonian system associated with the problem (OCP) as $\varepsilon$ tends to 0 . This convergence clearly holds as well for the solutions of the linearized system associated with the Hamiltonian system for $(\mathbf{O C P})_{\varepsilon}$; therefore, as $\varepsilon$ tends to $0, \frac{\partial \exp ^{\varepsilon}}{\partial x_{0}}\left(t, x_{0}, \alpha^{\varepsilon^{\prime}}\left(x_{0}\right)\right)$ (respectively, $\left.\frac{\partial \exp ^{\varepsilon}}{\partial p_{0}}\left(t, x_{0}, \alpha^{\varepsilon^{\prime}}\left(x_{0}\right)\right)\right)$ converges to $\frac{\partial \exp }{\partial x_{0}}\left(t, x_{0}, \alpha^{\prime}\left(x_{0}\right)\right)$ (respectively, $\left.\frac{\partial \exp }{\partial p_{0}}\left(t, x_{0}, \alpha^{\prime}\left(x_{0}\right)\right)\right)$ uniformly on $[0, t]$.

In what follows, the notation $\tau_{j}^{+}\left(x_{0}\right)$ (resp. $\tau_{j}^{-}\left(x_{0}\right)$ ) stands for the right limit (resp. the left limit). For $x_{0} \in O$ and $j=1, \ldots, s$, we call the jump of $\frac{\partial \exp }{\partial x_{0}}\left(t, x_{0}\right)$ at $\tau_{j}\left(x_{0}\right)$ the difference

$$
\frac{\partial \exp }{\partial x_{0}}\left(\tau_{j}^{+}\left(x_{0}\right), x_{0}\right)-\frac{\partial \exp }{\partial x_{0}}\left(\tau_{j}^{-}\left(x_{0}\right), x_{0}\right),
$$

which is, according to [33, Equation 3.10, p. 123] given by

$$
\begin{aligned}
& \frac{\partial \exp }{\partial x_{0}}\left(\tau_{j}^{+}\left(x_{0}\right), x_{0}\right)-\frac{\partial \exp }{\partial x_{0}}\left(\tau_{j}^{-}\left(x_{0}\right), x_{0}\right) \\
& =\left(u_{1}\left(\tau_{j}^{+}\left(x_{0}\right), x_{0}\right)-u_{1}\left(\tau_{j}^{-}\left(x_{0}\right), x_{0}\right)\right) Y_{1}\left(x\left(\tau_{1}\left(x_{0}\right), x_{0}\right)\right) \frac{\partial \tau_{j}}{\partial x_{0}}\left(x_{0}\right) \\
& =\left(\operatorname{sign}\left(\varphi_{1}\left(\tau_{j}^{+}\right)\right)-\operatorname{sign}\left(\varphi_{1}\left(\tau_{j}^{-}\right)\right)\right) Y_{1}\left(x\left(\tau_{j}\left(x_{0}\right), x_{0}\right)\right) \frac{\partial \tau_{j}}{\partial x_{0}}\left(x_{0}\right) .
\end{aligned}
$$

Due to this jump condition one cannot expect to get a $C^{1}$ convergence result on the whole interval. We will next estimate the difference

$$
\frac{\partial \exp ^{\varepsilon}}{\partial x_{0}}\left(\tau_{j}^{\varepsilon}\left(x_{0}\right)+\eta, x_{0}\right)-\frac{\partial \exp ^{\varepsilon}}{\partial x_{0}}\left(\tau_{j}^{\varepsilon}\left(x_{0}\right)-\eta, x_{0}\right),
$$

for $\eta>0$ small, and show that it converges to (13), whenever $\varepsilon$ tends to 0 , and then $\eta$ tends to 0 .

Lemma 2.2. There holds

$$
\begin{array}{r}
\lim _{\eta \rightarrow 0} \lim _{\varepsilon \rightarrow 0}\left(\frac{\partial \exp ^{\varepsilon}}{\partial x_{0}}\left(\tau_{1}^{\varepsilon}\left(x_{0}\right)+\eta, x_{0}\right)-\frac{\partial \exp ^{\varepsilon}}{\partial x_{0}}\left(\tau_{1}^{\varepsilon}\left(x_{0}\right)-\eta, x_{0}\right)\right) \\
=\frac{\partial \exp }{\partial x_{0}}\left(\tau_{1}^{+}\left(x_{0}\right), x_{0}\right)-\frac{\partial \exp }{\partial x_{0}}\left(\tau_{1}^{-}\left(x_{0}\right), x_{0}\right) .
\end{array}
$$


Proof. One has

$$
\begin{aligned}
& \frac{\partial}{\partial t}\left(\frac{\partial x^{\varepsilon}}{\partial x_{0}}\left(t, x_{0}\right)\right)=\left(\frac{\partial X}{\partial x_{0}}\left(x^{\varepsilon}\left(t, x_{0}\right)\right)+u_{1}^{\varepsilon}\left(t, x_{0}\right) \frac{\partial Y_{1}}{\partial x_{0}}\left(x^{\varepsilon}\left(t, x_{0}\right)\right)\right. \\
& \left.+\varepsilon \sum_{i=2}^{m} u_{i}^{\varepsilon}\left(t, x_{0}\right) \frac{\partial Y_{i}}{\partial x_{0}}\left(x^{\varepsilon}\left(t, x_{0}\right)\right)\right) \frac{\partial x^{\varepsilon}}{\partial x_{0}}\left(t, x_{0}\right) \\
& +Y_{1}\left(x^{\varepsilon}\left(t, x_{0}\right)\right) \frac{\partial u_{1}^{\varepsilon}}{\partial x_{0}}\left(t, x_{0}\right)+\varepsilon \sum_{i=2}^{m} Y_{i}\left(x^{\varepsilon}\left(t, x_{0}\right)\right) \frac{\partial u_{i}^{\varepsilon}}{\partial x_{0}}\left(t, x_{0}\right) \\
& \frac{\partial x^{\varepsilon}}{\partial x_{0}}\left(\tau_{1}^{\varepsilon}\left(x_{0}\right)+\eta, x_{0}\right)-\frac{\partial x^{\varepsilon}}{\partial x_{0}}\left(\tau_{1}^{\varepsilon}\left(x_{0}\right)-\eta, x_{0}\right) \\
& =\int_{\tau_{1}^{\varepsilon}\left(x_{0}\right)-\eta}^{\tau_{1}^{\varepsilon}\left(x_{0}\right)+\eta}\left(\frac{\partial X}{\partial x_{0}}\left(x^{\varepsilon}\left(t, x_{0}\right)\right)+u_{1}^{\varepsilon}\left(t, x_{0}\right) \frac{\partial Y_{1}}{\partial x_{0}}\left(x^{\varepsilon}\left(t, x_{0}\right)\right)\right. \\
& \left.+\varepsilon \sum_{i=2}^{m} u_{i}^{\varepsilon}\left(t, x_{0}\right) \frac{\partial Y_{i}}{\partial x_{0}}\left(x^{\varepsilon}\left(t, x_{0}\right)\right)\right) \frac{\partial x^{\varepsilon}}{\partial x_{0}}\left(t, x_{0}\right) d t \\
& +\int_{\tau_{1}^{\varepsilon}\left(x_{0}\right)-\eta}^{\tau_{1}^{\varepsilon}\left(x_{0}\right)+\eta} Y_{1}\left(x^{\varepsilon}\left(t, x_{0}\right)\right) \frac{\partial u_{1}^{\varepsilon}}{\partial x_{0}}\left(t, x_{0}\right) d t \\
& +\int_{\tau_{1}^{\varepsilon}\left(x_{0}\right)-\eta}^{\tau_{1}^{\varepsilon}\left(x_{0}\right)+\eta} \varepsilon \sum_{i=2}^{m} Y_{i}\left(x^{\varepsilon}\left(t, x_{0}\right)\right) \frac{\partial u_{i}^{\varepsilon}}{\partial x_{0}}\left(t, x_{0}\right) d t .
\end{aligned}
$$

It is easy to see that the limit when $\eta$ tends to zero of the limit when $\varepsilon$ tends to zero of the first and third term of the right side of the last equation is equal to zero. Only the limit term

$$
\lim _{\eta \rightarrow 0} \lim _{\varepsilon \rightarrow 0} \int_{\tau_{1}^{\varepsilon}\left(x_{0}\right)-\eta}^{\tau_{1}^{\varepsilon}\left(x_{0}\right)+\eta} Y_{1}\left(x^{\varepsilon}\left(t, x_{0}\right)\right) \frac{\partial u_{1}^{\varepsilon}}{\partial x_{0}}\left(t, x_{0}\right) d t
$$

deserves a special attention. Let us denote

$$
\varphi_{i}^{\varepsilon}\left(t, x_{0}\right)=\left\langle p^{\varepsilon}\left(t, x_{0}\right), Y_{i}\left(x^{\varepsilon}\left(t, x_{0}\right)\right)\right\rangle, i=1, \ldots, m .
$$

From (12), we compute easily

$$
\begin{aligned}
& \frac{\partial u_{1}^{\varepsilon}}{\partial x_{0}}\left(t, x_{0}\right)= \\
& \frac{\varepsilon^{2}\left(\frac{\partial \varphi_{1}^{\varepsilon}}{\partial x_{0}}\left(t, x_{0}\right) \sum_{i=2}^{m} \varphi_{i}^{\varepsilon}\left(t, x_{0}\right)^{2}-\varphi_{1}^{\varepsilon}\left(t, x_{0}\right) \sum_{i=2}^{m} \varphi_{i}^{\varepsilon}\left(t, x_{0}\right) \frac{\partial \varphi_{i}^{\varepsilon}}{\partial x_{0}}\left(t, x_{0}\right)\right)}{\left(\varphi_{1}^{\varepsilon}\left(t, x_{0}\right)^{2}+\varepsilon^{2} \sum_{i=2}^{m} \varphi_{i}^{\varepsilon}\left(t, x_{0}\right)^{2}\right)^{3 / 2}} .
\end{aligned}
$$

We will consider asymptotic expansions of these quantities around $\tau_{1}^{\varepsilon}\left(x_{0}\right)$. Since $\varphi_{1}^{\varepsilon}\left(\tau_{1}^{\varepsilon}\left(x_{0}\right), x_{0}\right)=0$ for every $x_{0}$, it follows that

$$
\frac{\partial \varphi_{1}^{\varepsilon}}{\partial x_{0}}\left(\tau_{1}^{\varepsilon}\left(x_{0}\right), x_{0}\right)=-\frac{\partial \varphi_{1}^{\varepsilon}}{\partial t}\left(\tau_{1}^{\varepsilon}\left(x_{0}\right), x_{0}\right) \frac{\partial \tau_{1}^{\varepsilon}}{\partial x_{0}}\left(x_{0}\right) .
$$

In what follows, denote $\tau_{1}^{\varepsilon}=\left(\tau_{1}^{\varepsilon}\left(x_{0}\right), x_{0}\right)$. One has

$$
\begin{aligned}
& \int_{\tau_{1}^{\varepsilon}-\eta}^{\tau_{1}^{\varepsilon}+\eta} Y_{1}^{\varepsilon}\left(x^{\epsilon}\left(t, x_{0}\right)\right) \frac{\partial u_{1}^{\varepsilon}}{\partial x_{0}}\left(t, x_{0}\right) d t \\
& =\int_{\tau_{1}^{\varepsilon}-\eta}^{\tau_{1}^{\varepsilon}+\eta}\left(Y_{1}\left(x^{\varepsilon}\left(\tau_{1}^{\varepsilon}\right)\right)+O\left(t-\tau_{1}^{\varepsilon}\right)\right) \\
& \quad \varepsilon^{2}\left(\frac{\partial \varphi_{1}^{\varepsilon}}{\partial x_{0}}\left(\tau_{1}^{\varepsilon}\right)+O\left(t-\tau_{1}^{\varepsilon}\right)\right) \sum_{i=2}^{m}\left(\varphi_{i}^{\varepsilon}\left(\tau_{1}^{\varepsilon}\right)+O\left(t-\tau_{1}^{\varepsilon}\right)\right)^{2} \\
& D \\
& -\frac{\varepsilon^{2}\left(\varphi_{1}^{\varepsilon}\left(\tau_{1}^{\varepsilon}\right)+O\left(t-\tau_{1}^{\varepsilon}\right)\right) \sum_{i=2}^{m}\left(\varphi_{i}^{\varepsilon}\left(\tau_{1}^{\varepsilon}\right)+O\left(t-\tau_{1}^{\varepsilon}\right)\right)}{D} \\
& \left.-\frac{\left(\frac{\partial \varphi_{i}^{\varepsilon}}{\partial x_{0}}\left(\tau_{1}^{\varepsilon}\right)+O\left(t-\tau_{1}^{\varepsilon}\right)\right)}{D}\right] d t
\end{aligned}
$$

where

$$
\begin{aligned}
D & =\left(\left(\frac{\partial \varphi_{1}^{\varepsilon}}{\partial t}\left(\tau_{1}^{\varepsilon}\right)\left(t-\tau_{1}^{\varepsilon}\right)+o\left(t-\tau_{1}^{\varepsilon}\right)\right)^{2}\right. \\
& \left.+\varepsilon^{2} \sum_{i=2}^{m}\left(\varphi_{i}^{\varepsilon}\left(\tau_{1}^{\varepsilon}\right)+\frac{\partial \varphi_{i}^{\varepsilon}}{\partial t}\left(\tau_{1}^{\varepsilon}\right)\left(t-\tau_{1}^{\varepsilon}\right)+o\left(t-\tau_{1}^{\varepsilon}\right)\right)^{2}\right)^{3 / 2}
\end{aligned}
$$

and simplifying the expression (14) (the terms of order $O\left(\left(t-\tau_{1}^{\varepsilon}\right)^{k}\right)$ and $o\left(\left(t-\tau_{1}^{\varepsilon}\right)^{l}\right)$, with $k=2,3$ and $l=1,2,3$, are omitted) we get 
where

$$
\begin{aligned}
& N_{1}=\sum_{i=2}^{m}\left(\varphi_{i}^{\varepsilon}\left(\tau_{1}^{\varepsilon}\right)\right)^{2}, N_{2}=\sum_{i=2}^{m}\left(\frac{\partial \varphi_{i}^{\varepsilon}}{\partial t}\left(\tau_{1}^{\varepsilon}\right)\right)^{2}, \\
& N_{3}=2 \sum_{i=2}^{m} \varphi_{i}^{\varepsilon}\left(\tau_{1}^{\varepsilon}\right) \frac{\partial \varphi_{i}^{\varepsilon}}{\partial t}\left(\tau_{1}^{\varepsilon}\right), \\
& M_{1}=2 \frac{\partial \varphi_{1}^{\varepsilon}}{\partial x_{0}}\left(\tau_{1}^{\varepsilon}\right) \sum_{i=2}^{m} \varphi_{i}^{\varepsilon}\left(\tau_{1}^{\varepsilon}\right)+\sum_{i=2}^{m}\left(\varphi_{i}^{\varepsilon}\left(\tau_{1}^{\varepsilon}\right)\right)^{2}, \\
& M_{2}=\sum_{i=2}^{m}\left(\frac{\partial \varphi_{i}^{\varepsilon}}{\partial x_{0}}\left(\tau_{1}^{\varepsilon}\right)\right)^{2} .
\end{aligned}
$$

Notice that the denominator never vanishes, since by Assumption 3 the functions $\left(t, x_{0}\right) \mapsto \varphi_{i}\left(t, x_{0}\right), i=$ $1, \ldots, m$ do not vanish simultaneously.

The limit when $\eta$ tends to zero of the limit when $\varepsilon$ tends to zero, of the first and second term of the right side of the last equality are respectively equal to $\left(\operatorname{sign}\left(\varphi_{1}\left(\tau_{1}^{+}\right)\right)-\operatorname{sign}\left(\varphi_{1}\left(\tau_{1}^{-}\right)\right)\right) Y_{1}\left(x\left(\tau_{1}\left(x_{0}\right), x_{0}\right)\right) \frac{\partial \tau_{1}}{\partial x_{0}}\left(x_{0}\right)$ and 0 . Since

$$
\lim _{\varepsilon \rightarrow 0} \frac{\partial \tau_{1}^{\varepsilon}}{\partial x_{0}}\left(x_{0}\right)=\frac{\partial \tau_{1}}{\partial x_{0}}\left(x_{0}\right)
$$

it follows that

$$
\begin{aligned}
& \lim _{\eta \rightarrow 0} \lim _{\varepsilon \rightarrow 0}\left(\frac{\partial x^{\varepsilon}}{\partial x_{0}}\left(\tau_{1}^{\varepsilon}\left(x_{0}\right)+\eta, x_{0}\right)-\frac{\partial x^{\varepsilon}}{\partial x_{0}}\left(\tau_{1}^{\varepsilon}\left(x_{0}\right)-\eta, x_{0}\right)\right) \\
& =\left(\operatorname{sign}\left(\varphi_{1}\left(\tau_{1}^{+}\right)\right)-\operatorname{sign}\left(\varphi_{1}\left(\tau_{1}^{-}\right)\right)\right) Y_{1}\left(x\left(\tau_{1}\left(x_{0}\right), x_{0}\right)\right) \frac{\partial \tau_{1}}{\partial x_{0}}\left(x_{0}\right),
\end{aligned}
$$

and the lemma follows.

A similar lemma holds for $\frac{\partial \exp }{\partial p_{0}}$. This result permits to extend the convergence result beyond the first switching time; the extension of Lemma 2.1 to every further interval $\left(\tau_{j-1}, \tau_{j}\right)$ is then straightforward. This proves the proposition.

We are now in a position to prove Theorem 6 . From Theorem 2, the trajectory $\hat{x}(\cdot)$ can be embedded into the field of extremals $x_{0} \mapsto \exp \left(t, x_{0}, \alpha^{\prime}\left(x_{0}\right)\right)$ with $x_{0} \in O$ and the mapping

$$
\begin{aligned}
\left(0, t_{c}\right) \times X & \rightarrow \mathbb{R}^{n} \\
\left(t, x_{0}\right) & \mapsto \exp \left(t, x_{0}, \alpha^{\prime}\left(x_{0}\right)\right)
\end{aligned}
$$

is of rank $n$, where $X=\left\{x_{0} \in\right.$ $\left.O \mid \max _{|w| \leqslant 1} H\left(x_{0}, \alpha^{\prime}\left(x_{0}\right), p^{0}, w\right)=0\right\}, O$ is a neighborhood of $\hat{x}_{0}$, and $t_{c}$ is the first conjugate time of $\hat{x}(\cdot)$.
From Remark 1.5, the determinants

$\operatorname{det}\left(\left.\frac{d}{d x_{0}} \exp \left(t, x_{0}, \alpha^{\prime}\left(x_{0}\right)\right)\right|_{\left(t, x_{0}\right) \in\left(\tau_{s}\left(x_{0}\right), \tau_{s+1}\left(x_{0}\right)\right) \times X}, f_{s+1}\left(x_{0}\right)\right)$

and

$\operatorname{det}\left(\left.\frac{d}{d x_{0}} \exp \left(t, x_{0}, \alpha^{\prime}\left(x_{0}\right)\right)\right|_{\left(t, x_{0}\right) \in\left(\tau_{s+1}\left(x_{0}\right), \tau_{s+2}\left(x_{0}\right)\right) \times X}, f_{s+2}\left(x_{0}\right)\right)$

have different signs, with $\tau_{s+1}\left(\hat{x}_{0}\right)=t_{c}$.

By Definition 1.4 , the point $x^{\varepsilon}\left(\tau_{c}^{\varepsilon}\left(x_{0}\right)\right)$ is geometrically conjugate to $x^{\varepsilon}(0)=x_{0}$, with $x_{0} \in X^{\varepsilon}$, if and only if

$$
\operatorname{det}\left(\frac{d}{d x_{0}} \exp ^{\varepsilon}\left(t, x_{0}, \alpha^{\varepsilon^{\prime}}\left(x_{0}\right)\right), f^{\varepsilon}\left(x_{0}\right)_{\mid x_{0} \in X^{\varepsilon}}\right)=0
$$

for $t=\tau_{c}^{\varepsilon}\left(x_{0}\right)$. Let $x_{0} \in X^{\varepsilon}$, we have

$$
\begin{aligned}
\frac{\partial \exp ^{\varepsilon}}{\partial x_{0}}\left(\tau^{\varepsilon}\left(x_{0}\right), x_{0}, \alpha^{\varepsilon^{\prime}}\left(x_{0}\right)\right) & \\
= & \frac{\partial \exp ^{\varepsilon}}{\partial t}\left(\tau^{\varepsilon}\left(x_{0}\right), x_{0}, \alpha^{\varepsilon^{\prime}}\left(x_{0}\right)\right) \frac{\partial \tau^{\varepsilon}}{\partial x_{0}}\left(x_{0}\right) \\
& +\frac{\partial \exp ^{\varepsilon}}{\partial x_{0}}\left(\tau^{\varepsilon}\left(x_{0}\right), x_{0}, \alpha^{\varepsilon^{\prime}}\left(x_{0}\right)\right) \\
& +\frac{\partial \exp ^{\varepsilon}}{\partial p_{0}}\left(\tau^{\varepsilon}\left(x_{0}\right), x_{0}, \alpha^{\varepsilon \prime}\left(x_{0}\right)\right) \alpha^{\varepsilon^{\prime \prime}}\left(x_{0}\right) .
\end{aligned}
$$

Since $\frac{\partial \exp ^{\varepsilon}}{\partial t}\left(\tau^{\varepsilon}\left(x_{0}\right), x_{0}, \alpha^{\varepsilon \prime}\left(x_{0}\right)\right)=\dot{x}^{\varepsilon}\left(x_{0}\right)=f^{\varepsilon}\left(x_{0}\right)$, there holds, clearly,

$$
\operatorname{det}\left(\frac{\partial \exp ^{\varepsilon}}{\partial t}\left(\tau^{\varepsilon}\left(x_{0}\right), x_{0}, \alpha^{\varepsilon^{\prime}}\left(x_{0}\right)\right) \frac{\partial \tau^{\varepsilon}}{\partial x_{0}}\left(x_{0}\right), f^{\varepsilon}\left(x_{0}\right)\right)=0,
$$

and it thus follows that

$$
\begin{aligned}
& \operatorname{det}\left(\frac{d}{d x_{0}} \exp ^{\varepsilon}\left(\tau^{\varepsilon}\left(x_{0}\right), x_{0}, \alpha^{\varepsilon^{\prime}}\left(x_{0}\right)\right), f^{\varepsilon}\left(x_{0}\right)\right) \\
& =\operatorname{det}\left(\frac{\partial \exp ^{\varepsilon}}{\partial x_{0}}\left(\tau^{\varepsilon}\left(x_{0}\right), x_{0}, \alpha^{\varepsilon^{\prime}}\left(x_{0}\right)\right)\right. \\
& \left.\quad+\frac{\partial \exp ^{\varepsilon}}{\partial p_{0}}\left(\tau^{\varepsilon}\left(x_{0}\right), x_{0}, \alpha^{\varepsilon^{\prime}}\left(x_{0}\right)\right) \alpha^{\varepsilon^{\prime \prime}}\left(x_{0}\right), f^{\varepsilon}\left(x_{0}\right)\right) \\
& =\operatorname{det}\left(\frac{d}{d x_{0}} \exp ^{\varepsilon}\left(t, x_{0}, \alpha^{\varepsilon \prime}\left(x_{0}\right)\right), f^{\varepsilon}\left(x_{0}\right)\right)
\end{aligned}
$$

for $t=\tau^{\varepsilon}\left(x_{0}\right)$. By Proposition 7, on every compact subinterval of $\left(\tau_{j-1}\left(x_{0}\right), \tau_{j}\left(x_{0}\right)\right)$, the mapping $\left(t, x_{0}\right) \mapsto \exp ^{\varepsilon}\left(t, x_{0}, \alpha^{\varepsilon^{\prime}}\left(x_{0}\right)\right)$ converges to $\left(t, x_{0}\right) \mapsto$ $\exp \left(t, x_{0}, \alpha^{\prime}\left(x_{0}\right)\right)$ uniformly in $C^{1}$ topology, therefore the determinants

$\operatorname{det}\left(\left.\frac{d}{d x_{0}} \exp ^{\varepsilon}\left(t, x_{0}, \alpha^{\varepsilon^{\prime}}\left(x_{0}\right)\right)\right|_{\left(t, x_{0}\right) \in\left(\tau_{s}^{\varepsilon}\left(x_{0}\right), \tau_{s+1}^{\varepsilon}\left(x_{0}\right)\right) \times X^{\varepsilon}}, f^{\varepsilon}\left(x_{0}\right)\right)$ 
and

$\operatorname{det}\left(\frac{d}{d x_{0}} \exp ^{\varepsilon}\left(t, x_{0},\left.\alpha^{\left.\varepsilon^{\prime}\left(x_{0}\right)\right)}\right|_{\left(t, x_{0}\right) \in\left(\tau_{s+1}^{\varepsilon}\left(x_{0}\right), \tau_{s+2}^{\varepsilon}\left(x_{0}\right)\right) \times X^{\varepsilon}}, f^{\varepsilon}\left(x_{0}\right)\right)\right.$

have different signs before and after $\tau_{s+1}^{\varepsilon}\left(x_{0}\right)$. Therefore, by continuity, the function $t \mapsto$ $\operatorname{det}\left(\frac{d}{d x_{0}} \exp ^{\varepsilon}\left(t, x_{0}, \alpha^{\varepsilon^{\prime}}\left(x_{0}\right)\right), f^{\varepsilon}\left(x_{0}\right)\right)$ vanishes for some time, close to $\tau_{s+1}^{\varepsilon}\left(x_{0}\right)$. By Definition 1.4, this time $t_{c}^{\varepsilon}\left(x_{0}\right)$ is a geometrically conjugate time, and when $\varepsilon$ tends to $0, t_{c}^{\varepsilon}\left(\hat{x}_{0}\right)$ converges to the bang-bang conjugate time $t_{c}=\tau_{s+1}\left(\hat{x}_{0}\right)$. This ends the proof of the theorem.

\section{Examples}

In this section we illustrate Theorem 6 with two examples of minimal time control problems.

\subsection{First example: the Rayleigh problem}

In this section we consider the minimal time control problem for the Rayleigh control system (see e.g. [25, 30]),

$$
\begin{aligned}
& \dot{x}_{1}(t)=x_{2}(t), \\
& \dot{x}_{2}(t)=-x_{1}(t)+x_{2}(t)\left(1.4-0.14 x_{2}(t)^{2}\right)+u_{1}(t),
\end{aligned}
$$

with the control constraint

$$
\left|u_{1}(\cdot)\right| \leqslant 4,
$$

and with boundary conditions given by

$$
x_{1}(0)=-4, x_{2}(0)=-3, x_{1}\left(t_{f}\right)=x_{2}\left(t_{f}\right)=0 .
$$

According to the Pontryagin maximum principle, any optimal solution $\hat{x}(\cdot)$ of (15)-(17) is the projection of an extremal $\left(\hat{x}(\cdot), \hat{p}(\cdot), p^{0}, \hat{u}_{1}(\cdot)\right)$ such that

$$
\begin{aligned}
& \dot{\hat{p}}_{1}(t)=\hat{p}_{2}(t) \\
& \dot{\hat{p}}_{2}(t)=-\hat{p}_{1}(t)-\hat{p}_{2}(t)\left(1.4-0.42 \hat{x}_{2}(t)^{2}\right)
\end{aligned}
$$

and the maximization condition $\hat{p}_{2}(t) \hat{u}_{1}(t)=$ $\max _{|w| \leqslant 4}\left(\hat{p}_{2}(t) w\right)$ holds almost everywhere on $\left[0, t_{f}\right]$. It is easy to see that $\hat{p}_{2}(\cdot)$ cannot vanish on some subinterval, and it follows that the optimal control $\hat{u}_{1}(\cdot)$ is bang-bang, equal to $\hat{u}_{1}(t)=4 \operatorname{sign}\left(\hat{p}_{2}(t)\right)$. Applying a shooting method to problem (15)-(17) (with $p^{0}=-1$ ), we determine the initial adjoint vector $\hat{p}(0) \simeq(0.53095052 ; 0.34206485)$, and observe that the trajectory has only one switching time $\hat{\tau}_{1} \simeq 0.57613128$ on $\left[0, t_{f}\right]$, that is, $\hat{u}_{1}(\cdot)$ is given by

$$
\hat{u}_{1}(t)= \begin{cases}+4 & \text { for } 0 \leqslant t \leqslant \hat{\tau}_{1} \\ -4 & \text { for } \hat{\tau}_{1} \leqslant t \leqslant t_{f}\end{cases}
$$

with a final time $t_{f} \simeq 2.97812917$ (see Figures 3-4). Furthermore, $\hat{x}(\cdot)$ is the unique minimal time solution and has a unique extremal lift (up to a multiplicative scalar), which is moreover normal.

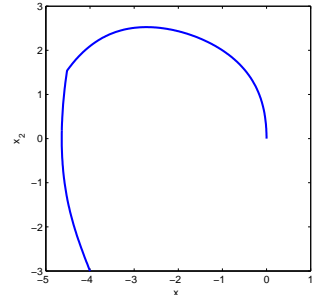

Figure 3: Optimal trajectory

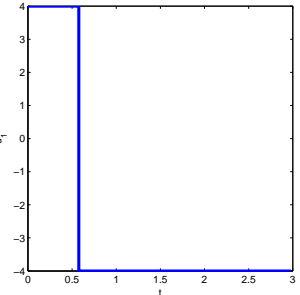

Figure 4: Optimal control
Prolongating the trajectory $\hat{x}(\cdot)$ to the interval $[0,4]$, we observe a second switching time at $\hat{\tau}_{2} \simeq$ 3.14750955 .

Notice that the second-order sufficient conditions of $[27,28,29,30]$ are satisfied before $\hat{\tau}_{2}$, confirming the local optimality status of the trajectory, but are no longer satisfied beyond this second switching time; we can thus expect the trajectory not to be locally optimal beyond $\hat{\tau}_{2}$. To investigate this optimality status we use the extremal field approach.

From Theorem 2 and Remark 1.4, the first conjugate point $\hat{x}\left(t_{c}\right)$ is an overlap point of the extremal field emanating from the horizontal one-dimensional manifold $X=\left\{x_{0} \in O \mid \max _{|w| \leqslant 1} H\left(x_{0}, \alpha^{\prime}\left(x_{0}\right),-1, w\right)=0\right\}$. In practice, the function $\alpha$ is not known, and we rather use the field of extremals emanating from the vertical manifold $X_{p}=\left\{p_{0} \in O_{p} \mid \max _{|w| \leqslant 1} H\left(\hat{x}_{0}, p_{0},-1, w\right)=0\right\}$ (see $[11,38]$ ), where $O_{p}$ is a neighborhood of the initial value of the adjoint vector $\hat{p}(0)$. The characterization in terms of fold point still holds for this vertical manifold (see [38]). We observe on Figures 5-6 that this field of extremals reflects off the switching surface at the second switching time; the point $\hat{x}\left(\hat{\tau}_{2}\right)$ is a fold point and the first conjugate time is equal to the second switching time, $t_{c}=\hat{\tau}_{2} \simeq 3.14750955$.

We next propose a regularization procedure, for which we compute the first geometric conjugate time $t_{c}^{\varepsilon}$ and check that it indeed converges to the first conjugate time $t_{c}$ of the bang-bang case as $\varepsilon$ tends to 0 .

We consider the regularized control system

$$
\begin{aligned}
& \dot{x}_{1}^{\varepsilon}(t)=x_{2}^{\varepsilon}(t)+\varepsilon u_{2}^{\varepsilon}(t), \\
& \dot{x}_{2}^{\varepsilon}(t)=-x_{1}^{\varepsilon}(t)+x_{2}^{\varepsilon}(t)\left(1.4-0.14 x_{2}^{\varepsilon}(t)^{2}\right)+u_{1}^{\varepsilon}(t),
\end{aligned}
$$

with the boundary conditions (17), and where the con- 


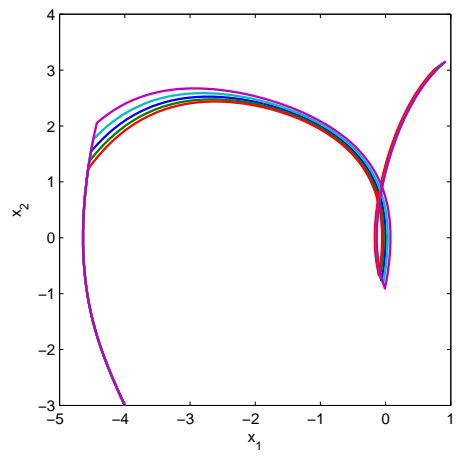

Figure 5: Extremal field $t \in[0,4]$

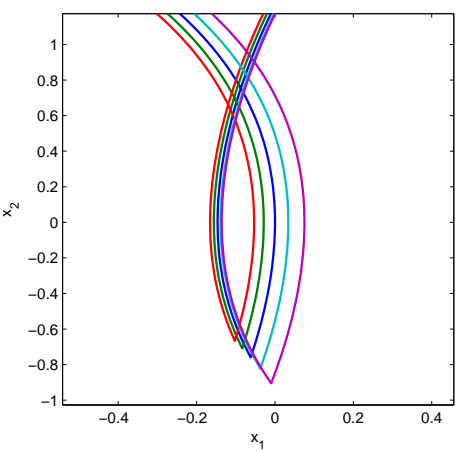

Figure 6: Overlap of the flow

trol $u^{\varepsilon}(\cdot)=\left(u_{1}^{\varepsilon}(\cdot), u_{2}^{\varepsilon}(\cdot)\right)$ satisfies the constraint

$$
\left(u_{1}^{\varepsilon}(\cdot)\right)^{2}+\left(u_{2}^{\varepsilon}(\cdot)\right)^{2} \leqslant 16 .
$$

Any optimal solution $\hat{x}^{\varepsilon}(\cdot)$ of (17)-(20) is the projection of an extremal $\left(\hat{x}^{\varepsilon}(\cdot), \hat{p}^{\varepsilon}(\cdot), p^{0 \varepsilon}, \hat{u}^{\varepsilon}(\cdot)\right)$ such that

$$
\begin{aligned}
& \dot{\hat{p}}_{1}^{\varepsilon}(t)=\hat{p}_{2}^{\varepsilon}(t) \\
& \dot{\hat{p}}_{2}^{\varepsilon}(t)=-\hat{p}_{1}^{\varepsilon}(t)-\hat{p}_{2}^{\varepsilon}(t)\left(1.4-0.42 \hat{x}_{2}^{\varepsilon}(t)^{2}\right) .
\end{aligned}
$$

The Assumption 3 is verified, and the controls that satisfy the Pontryagin maximization condition (11) are given by

$$
\begin{aligned}
& \hat{u}_{1}^{\varepsilon}(t)=\frac{4 \hat{p}_{2}^{\varepsilon}(t)}{\sqrt{\left(\hat{p}_{2}^{\varepsilon}(t)\right)^{2}+\varepsilon^{2}\left(\hat{p}_{1}^{\varepsilon}(t)\right)^{2}}}, \\
& \hat{u}_{2}^{\varepsilon}(t)=\frac{4 \varepsilon \hat{p}_{1}^{\varepsilon}(t)}{\sqrt{\left(\hat{p}_{2}^{\varepsilon}(t)\right)^{2}+\varepsilon^{2}\left(\hat{p}_{1}^{\varepsilon}(t)\right)^{2}}} .
\end{aligned}
$$

Applying a shooting method to this problem, we determine the optimal trajectory of the regularized problem, and we indeed observe the expected convergence of $\left(\hat{x}^{\varepsilon}(\cdot), \hat{p}^{\varepsilon}(\cdot),-1, \hat{u}^{\varepsilon}\right)$ towards $\left(\hat{x}(\cdot), \hat{p}(\cdot),-1, \hat{u}_{1}\right)$, as $\varepsilon$ tends to 0 , in agreement with Theorem 4 (see Figures 79).

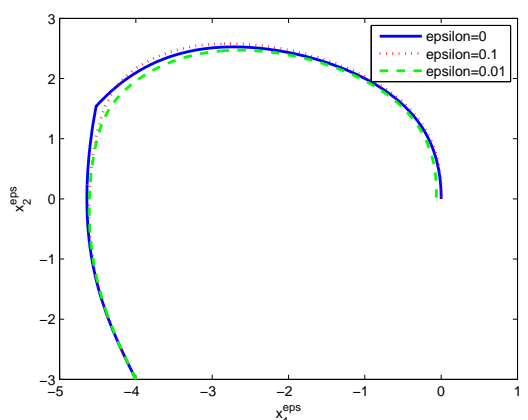

Figure 7: Trajectory
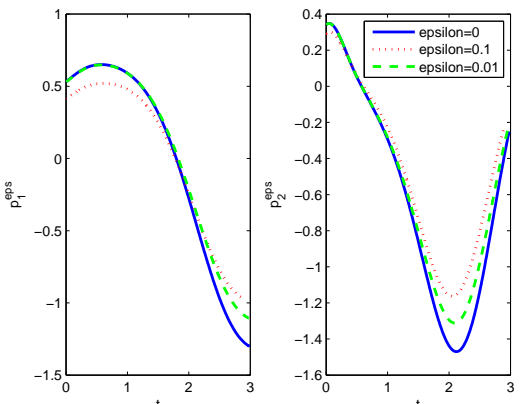

Figure 8: Adjoint vector

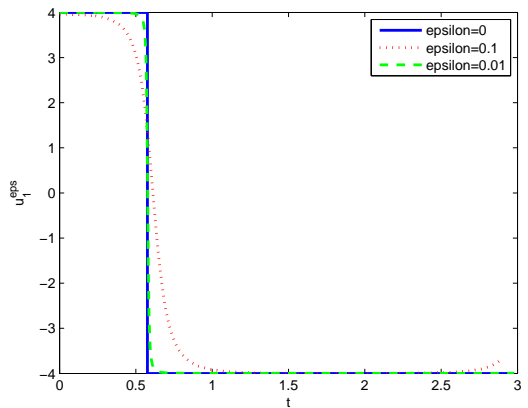

Figure 9: Control

The optimal controls (21) are smooth functions of $t$, therefore the algorithms presented in [11] to compute the first conjugate time along a smooth extremal curve 
can be applied. Here we will apply the test for conjugate times explained in [11] when the final time is free and the extremal is normal. Let us briefly recall this test. The maximized Hamiltonian writes as

$H_{r}^{\varepsilon}\left(\hat{x}^{\varepsilon}, \hat{p}^{\varepsilon}\right)=\hat{p}_{1}^{\varepsilon}\left(\hat{x}_{2}^{\varepsilon}+\frac{4 \varepsilon^{2} \hat{p}_{1}^{\varepsilon}}{\sqrt{\left(\hat{p}_{2}^{\varepsilon}\right)^{2}+\varepsilon^{2}\left(\hat{p}_{1}^{\varepsilon}\right)^{2}}}\right)$

$+\hat{p}_{2}^{\varepsilon}\left(-\hat{x}_{1}^{\varepsilon}+\hat{x}_{2}^{\varepsilon}\left(1.4-0.14\left(\hat{x}_{2}^{\varepsilon}\right)^{2}\right)+\frac{4 \hat{p}_{2}^{\varepsilon}}{\sqrt{\left(\hat{p}_{2}^{\varepsilon}\right)^{2}+\varepsilon^{2}\left(\hat{p}_{1}^{\varepsilon}\right)^{2}}}\right)-1$.

The aim is to compute the solution $Z^{\varepsilon}(\cdot)=$ $\left(\delta x_{1}^{\varepsilon}(\cdot), \delta x_{2}^{\varepsilon}(\cdot), \delta p_{1}^{\varepsilon}(\cdot), \delta p_{2}^{\varepsilon}(\cdot)\right)^{T}$ of the so-called variational system $\dot{Z}^{\varepsilon}(t)=V(t) Z^{\varepsilon}(t)$ along the extremal $\left(\hat{x}^{\varepsilon}(\cdot), \hat{p}^{\varepsilon}(\cdot)\right)$, where

$$
V(t)=\left(\begin{array}{cc}
\frac{\partial^{2} H_{r}^{\varepsilon}}{\partial x \partial p}\left(\hat{x}^{\varepsilon}(t), \hat{p}^{\varepsilon}(t)\right) & \frac{\partial^{2} H_{r}^{\varepsilon}}{\partial p^{2}}\left(\hat{x}^{\varepsilon}(t), \hat{p}^{\varepsilon}(t)\right) \\
-\frac{\partial^{2} H_{r}^{\varepsilon}}{\partial x^{2}}\left(\hat{x}^{\varepsilon}(t), \hat{p}^{\varepsilon}(t)\right) & -\frac{\partial^{2} H_{r}^{\varepsilon}}{\partial x \partial p}\left(\hat{x}^{\varepsilon}(t), \hat{p}^{\varepsilon}(t)\right)
\end{array}\right)
$$

with initial conditions $\left(\delta x_{1}^{\varepsilon}(0), \delta x_{2}^{\varepsilon}(0)\right)=(0,0)$ and $\left(\delta p_{1}^{\varepsilon}(0), \delta p_{2}^{\varepsilon}(0)\right)$ such that the scalar product $\left\langle\left(f_{1}^{\varepsilon}(0), f_{2}^{\varepsilon}(0)\right),\left(\delta p_{1}^{\varepsilon}(0), \delta p_{2}^{\varepsilon}(0)\right)\right\rangle$ is equal to 0 , where $\left(f_{1}^{\varepsilon}, f_{2}^{\varepsilon}\right)$ is the dynamics, given by

$\left\{\begin{array}{l}f_{1}^{\varepsilon}(t)=x_{2}^{\varepsilon}(t)+\frac{4 \varepsilon^{2} p_{1}^{\varepsilon}(t)}{\sqrt{\left(p_{2}^{\varepsilon}(t)\right)^{2}+\varepsilon^{2}\left(p_{1}^{\varepsilon}(t)\right)^{2}}}, \\ f_{2}^{\varepsilon}(t)=-x_{1}^{\varepsilon}(t)+x_{2}^{\varepsilon}(t)\left(1.4-0.14 x_{2}^{\varepsilon}(t)^{2}\right)+\frac{4 p_{2}^{\varepsilon}(t)}{\sqrt{\left(p_{2}^{\varepsilon}(t)\right)^{2}+\varepsilon^{2}\left(p_{1}^{\varepsilon}(t)\right)^{2}}} .\end{array}\right.$

The first geometric conjugate time is then the first positive zero of the function $t \mapsto$ $\operatorname{det}\left(\delta x_{1}^{\varepsilon}(t) \delta x_{2}^{\varepsilon}(t), f_{1}^{\varepsilon}(t) f_{2}^{\varepsilon}(t)\right)$ (see Figure 10).

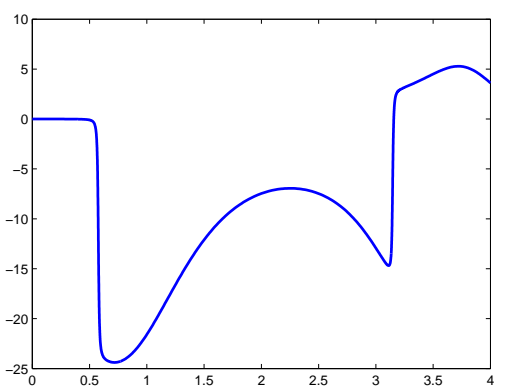

Figure 10: Determinant $(\varepsilon=0.01)$

We report on Table 3.1 the values of the first geometric conjugate time of the optimal trajectory $\hat{x}^{\varepsilon}(\cdot)$, for different values of $\varepsilon$. We observe that, as expected, $t_{c}^{\varepsilon}$ converges to $t_{c} \simeq 3.14750955$ as $\varepsilon$ tends to 0 .

\begin{tabular}{|l|l|}
\hline$\varepsilon$ & $t_{c}^{\varepsilon}$ \\
\hline 0.1 & 3.26735859 \\
0.01 & 3.1559626 \\
0.001 & 3.14844987 \\
0.0001 & 3.14760515 \\
\hline
\end{tabular}

Table 1: Values of $t_{c}^{\varepsilon}$

Another possible test (see [11]) is to compute numerically solutions $Z_{i}(\cdot)=\left(\delta x_{1 i}^{\varepsilon}(\cdot), \delta x_{2 i}^{\varepsilon}(\cdot), \delta p_{1 i}^{\varepsilon}(\cdot), \delta p_{2 i}^{\varepsilon}(\cdot)\right)$, $i=1,2$, of the variational system considered previously, with initial conditions $\left(\delta p_{11}^{\varepsilon}(0), \delta p_{21}^{\varepsilon}(0)\right)=(1,0)$ and $\left(\delta p_{12}^{\varepsilon}(0), \delta p_{22}^{\varepsilon}(0)\right)=(0,1)$, and then to compute the rank of the matrix

$$
J^{\varepsilon}(t)=\left(\begin{array}{ll}
\delta x_{11}^{\varepsilon}(t) & \delta x_{21}^{\varepsilon}(t) \\
\delta x_{12}^{\varepsilon}(t) & \delta x_{22}^{\varepsilon}(t)
\end{array}\right)
$$

This rank must be equal to 1 outside a conjugate time, and 0 at a conjugate time. In order to compute it, we use a singular value decomposition of $J^{\varepsilon}(t)$; then, a conjugate time occurs whenever the first singular value of $J^{\varepsilon}(t)$ vanishes (see Figure 11).

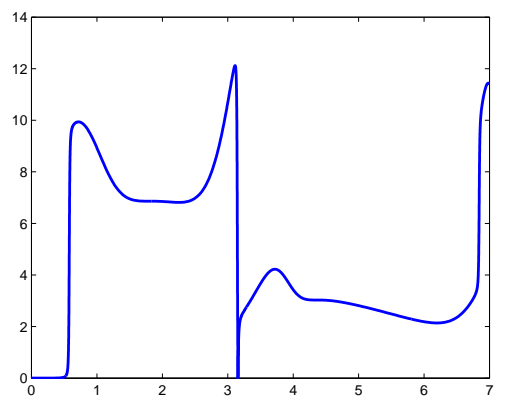

Figure 11: First singular value of $J^{\varepsilon}(t)(\varepsilon=0.01)$

In this first example, the first conjugate time $t_{c}$ of the optimal bang-bang trajectory $\hat{x}(\cdot)$ coincides with the second switching time. We next provide an example where the first conjugate time is equal to the third switching time.

\subsection{Second example}

Consider the minimal time control problem for the control system

$$
\begin{aligned}
& \dot{x}_{1}(t)=\sin \left(x_{2}(t)\right), \\
& \dot{x}_{2}(t)=-\sin \left(x_{1}(t)\right)+u_{1}(t),
\end{aligned}
$$


with the control constraint

$$
\left|u_{1}(\cdot)\right| \leqslant 1
$$

and with the boundary conditions

$$
x_{1}(0)=x_{2}(0)=0, x_{1}\left(t_{f}\right)=2.9, x_{2}\left(t_{f}\right)=0.1 .
$$

From the Pontryagin maximum principle, any optimal solution $\hat{x}(\cdot)$ of $(22)-(24)$ is the projection of an extremal $\left(\hat{x}(\cdot), \hat{p}(\cdot), p^{0}, \hat{u}_{1}(\cdot)\right)$ such that

$$
\begin{aligned}
& \dot{\hat{p}}_{1}(t)=\hat{p}_{2}(t) \cos \left(\hat{x}_{1}(t)\right), \\
& \dot{\hat{p}}_{2}(t)=-\hat{p}_{1}(t) \cos \left(\hat{x}_{2}(t)\right),
\end{aligned}
$$

and the maximization condition $\hat{p}_{2}(t) \hat{u}_{1}(t)=$ $\max _{|w| \leqslant 1}\left(\hat{p}_{2}(t) w\right)$ must hold almost everywhere on $\left[0, t_{f}\right]$. It is easy to see that $\hat{p}_{2}(\cdot)$ cannot vanish on some subinterval, and it follows that the optimal control $\hat{u}_{1}(\cdot)$ is bang-bang, equal to $\hat{u}_{1}(t)=\operatorname{sign}\left(\hat{p}_{2}(t)\right)$. Applying a shooting method to problem (22)-(24) (with $p^{0}=-1$ ), we determine the initial adjoint vector $\hat{p}(0)=(-0.5,1)$, and observe that the trajectory has one switching time $\hat{\tau}_{1} \simeq 3.26174615$ on $\left[0, t_{f}\right]$, that is, $\hat{u}_{1}(\cdot)$ is given by

$$
\hat{u}_{1}(t)= \begin{cases}+1 & \text { for } 0 \leqslant t \leqslant \hat{\tau}_{1}, \\ -1 & \text { for } \hat{\tau}_{1} \leqslant t \leqslant t_{f},\end{cases}
$$

with a final time $t_{f} \simeq 4.07756604$ (see Figures 12-13). Furthermore, $\hat{x}(\cdot)$ is the unique minimal time solution and has a unique extremal lift (up to a multiplicative scalar), which is moreover normal.

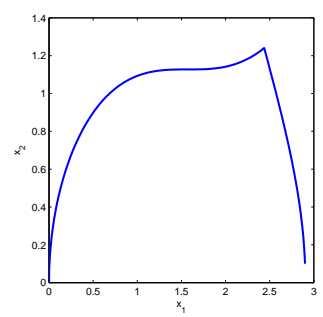

Figure 12: Optimal trajectory

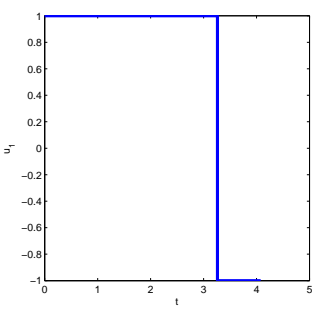

Figure 13: Optimal control
Prolongating the trajectory $\hat{x}(\cdot)$ to the interval $[0,11]$, we observe a second switching time at $\hat{\tau}_{2} \simeq$ 6.21787838 , and a third one at $\hat{\tau}_{3} \simeq 10.46930198$. Considering as in the previous example the extremal field emanating from the vertical manifold, we observe on Figures 14-15 that the extremal field crosses transversally the second switching surface, but reflects off the third switching surface, and it follows from Theorem 2 that the first conjugate time $t_{c}$ is equal to $\hat{\tau}_{3}$.

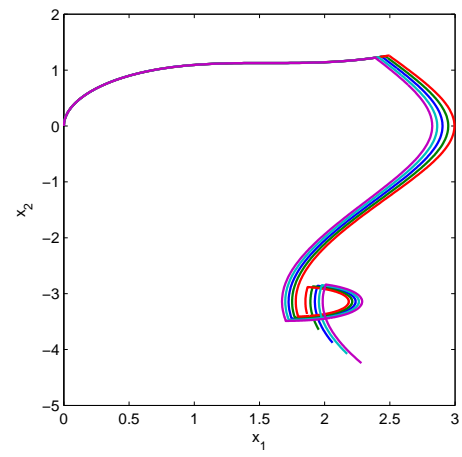

Figure 14: Extremal field, $t \in[0,11]$

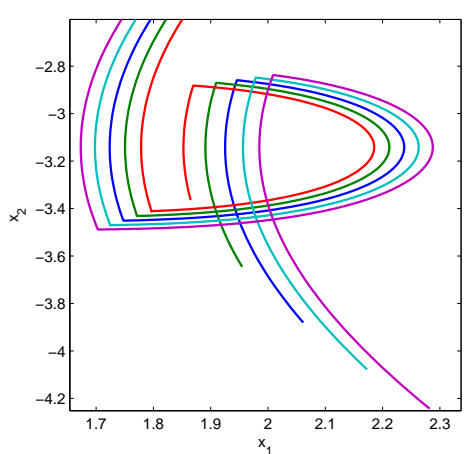

Figure 15: Zoom on the overlap of the flow at the third switching time

We propose the following regularization. Consider the control system

$$
\begin{aligned}
& \dot{x}_{1}^{\varepsilon}(t)=\sin \left(x_{2}^{\varepsilon}(t)\right)+\varepsilon u_{2}^{\varepsilon}(t), \\
& \dot{x}_{2}^{\varepsilon}(t)=-\sin \left(x_{1}^{\varepsilon}(t)\right)+u_{1}^{\varepsilon}(t),
\end{aligned}
$$

with the control constraint

$$
\left(u_{1}^{\varepsilon}(\cdot)\right)^{2}+\left(u_{2}^{\varepsilon}(\cdot)\right)^{2} \leqslant 1
$$

and the initial and final conditions (24). Any optimal solution $\hat{x}^{\varepsilon}(\cdot)$ of $(24)-(26)$ is the projection of an extremal $\left(\hat{x}^{\varepsilon}(\cdot), \hat{p}^{\varepsilon}(\cdot), p^{0^{\varepsilon}}, \hat{u}^{\varepsilon}(\cdot)\right)$ such that

$$
\begin{aligned}
& \dot{\hat{p}}_{1}^{\varepsilon}(t)=\hat{p}_{2}^{\varepsilon}(t) \cos \left(\hat{x}_{1}^{\varepsilon}(t)\right), \\
& \dot{\hat{p}}_{2}^{\varepsilon}(t)=-\hat{p}_{1}^{\varepsilon}(t) \cos \left(\hat{x}_{2}^{\varepsilon}(t)\right),
\end{aligned}
$$

and the maximization condition implies that the ex- 
tremal controls are given by

$$
\begin{aligned}
& \hat{u}_{1}^{\varepsilon}(t)=\frac{\hat{p}_{2}^{\varepsilon}(t)}{\sqrt{\left(\hat{p}_{2}^{\varepsilon}(t)\right)^{2}+\varepsilon^{2}\left(\hat{p}_{1}^{\varepsilon}(t)\right)^{2}}}, \\
& \hat{u}_{2}^{\varepsilon}(t)=\frac{\varepsilon \hat{p}_{1}^{\varepsilon}(t)}{\sqrt{\left(\hat{p}_{2}^{\varepsilon}(t)\right)^{2}+\varepsilon^{2}\left(\hat{p}_{1}^{\varepsilon}(t)\right)^{2}}} .
\end{aligned}
$$

Applying a shooting method to this problem, we determine the optimal trajectory of the regularized problem, and we indeed observe the expected convergence of $\left(\hat{x}^{\varepsilon}(\cdot), \hat{p}^{\varepsilon}(\cdot),-1, \hat{u}^{\varepsilon}\right)$ towards $\left(\hat{x}(\cdot), \hat{p}(\cdot),-1, \hat{u}_{1}\right)$, as $\varepsilon$ tends to 0 , in agreement with Theorem 4 (see Figures 16-18).

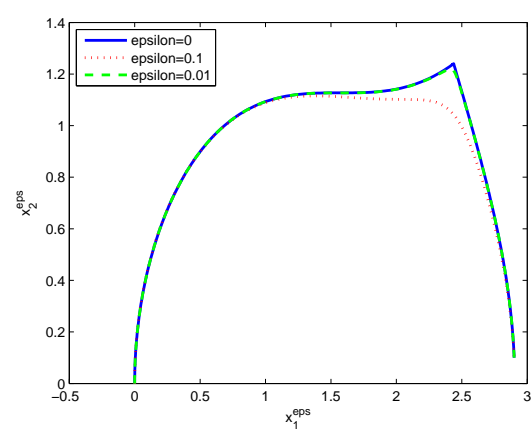

Figure 16: Trajectory
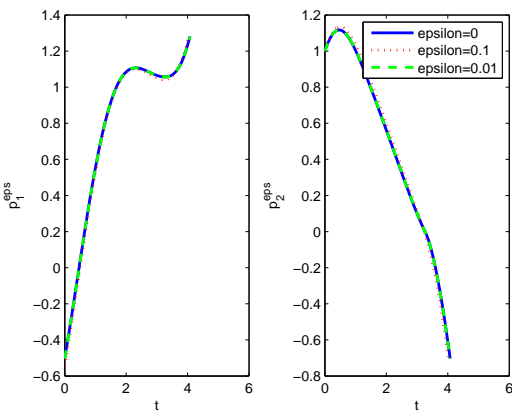

Figure 17: Adjoint vector

As in the previous example, the controls (27) are smooth functions of $t$, and we apply the algorithm described in [11], computing as before the determinant $\operatorname{det}\left(\delta x_{1}^{\varepsilon}(t) \delta x_{2}^{\varepsilon}(t), f_{1}^{\varepsilon}(t) f_{2}^{\varepsilon}(t)\right)$ (see Figure 19).

We report on Table 3.2 the values of the first geometric conjugate time of the optimal trajectory $\hat{x}^{\varepsilon}(\cdot)$, for different values of $\varepsilon$. We observe that, as expected, $t_{c}^{\varepsilon}$ converges to $t_{c}$ as $\varepsilon$ tends to 0 .

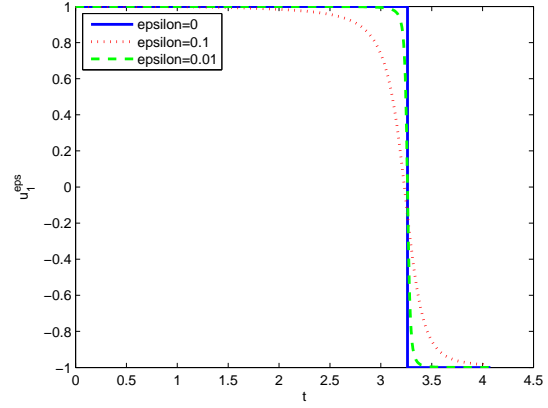

Figure 18: Control

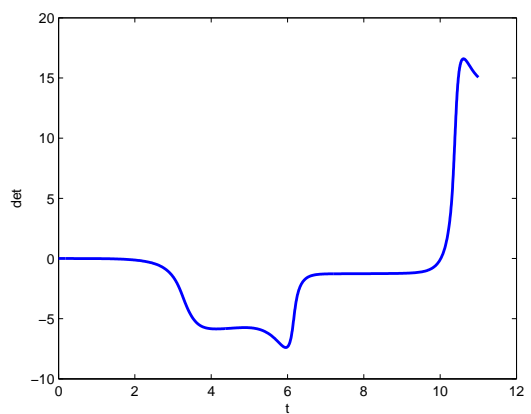

Figure 19: Determinant $(\varepsilon=0.1)$

Remark 3.1. We observe on both previous examples that it is not needed to consider very small values of $\varepsilon$ to estimate the first conjugate time $t_{c}$. Indeed, a conjugate time of a locally bang-bang trajectory can only occur at a switching time (see Remark 1.2) and, under our assumptions, switching times are isolated (see Remark 1.1). From Theorem 6, the first geometric conjugate time $t_{c}^{\varepsilon}$ converges to $t_{c}$, when $\varepsilon$ tend to 0 . Therefore, as soon as $\varepsilon$ is small enough so that $t_{c}^{\varepsilon}$ is in a (not necessarily so small) neighborhood of some switching time $\hat{\tau}_{s}$ of the bang-bang trajectory $\hat{x}(\cdot)$, this means that the bang-bang conjugate time $t_{c}$ is equal to that switching time $\hat{\tau}_{s}$.

\subsection{Conclusion}

In this article we focused on the problem of determining an efficient procedure to compute the first conjugate time $t_{c}$ for the minimal time problem for single-input control-affine systems $\dot{x}=X(x)+u_{1} Y_{1}(x)$ in $\mathbb{R}^{n}$ with the control constraint $\left|u_{1}(\cdot)\right| \leqslant 1$. We used the asymptotic approach developed in [44] which consists in adding new smooth vector fields $Y_{2}, \ldots, Y_{m}$ and a small param- 


\begin{tabular}{|l|l|}
\hline$\varepsilon$ & $t_{c}^{\varepsilon}$ \\
\hline 0.1 & 10.01593283 \\
0.01 & 10.3164905 \\
0.001 & 10.41858121 \\
0.0001 & 10.45291892 \\
0.00001 & 10.46419119 \\
\hline
\end{tabular}

Table 2: Values of $t_{c}^{\varepsilon}$

eter $\varepsilon>0$, so as to come up with the minimal time problem for the system $\dot{x}=X(x)+u_{1}^{\varepsilon} Y_{1}(x)+\varepsilon \sum_{i=2}^{m} u_{i}^{\varepsilon} Y_{i}(x)$, under the control constraint $\sum_{i=1}^{m}\left(u_{i}^{\varepsilon} \cdot(\cdot)\right)^{2} \leqslant 1$, with the same boundary conditions as the initial problem. Under appropriate assumptions, the optimal controls of the latter problem, depending on $\varepsilon$, are smooth functions of $t$, and the theoretical and practical results for the conjugate time theory that are well known in the smooth case can be applied to the regularized problem. We proved that the first conjugate time of regularized problem converges to the first conjugate time initial problem, when $\varepsilon$ tends to 0 . We thus get as a byproduct an efficient way to compute conjugate times in the bang-bang case. We provided examples to illustrate our result.

Note that our results still hold if the control-affine system is considered on a manifold. In this article we considered $\mathbb{R}^{n}$ for the sake of simplicity.

An open question is to extend our results to multiinput control-affine systems $\dot{x}=X(x)+\sum_{i=1}^{p} u_{i} Y_{i}(x)$ in $\mathbb{R}^{n}$, where $u=\left(u_{1}, \ldots, u_{p}\right) \in L^{\infty}\left(\left[0, t_{f}\right], \Delta\right)$ and $\Delta$ is a polyhedron (see [38]), or a convex polyhedron (see [30]), or a convex compact polyhedron (see [43]) of $\mathbb{R}^{p}$. For $p>1$, it would be interesting to consider the case where multiple switching times may occur, that is, when at least two control functions switch at the same time. A more general open question concerns the generalization to general cost functions, and/or more general dynamics.

Acknowledgment. We warmly thank Laura Poggiolini and Gianna Stefani for their help and remarks.

\section{References}

[1] A. Agrachev, R. Gamkrelidze, Symplectic methods for optimization and control, in: B. Jajubczyk, W. Respondek (Eds.), Geometry of Feedback and Optimal Control, Marcel Dekker, (1998), 19-78.

[2] A. Agrachev, G. Stefani, P. Zezza, A Hamitonian approach to strong minima in optimal control, in: Proceedings of Symposia in Pure Mathematics, Vol. 64, American Mathematical Society, (1998), 11-22.
[3] A. Agrachev, G. Stefani, P. Zezza, Strong optimality for a bang-bang trajectory, SIAM J. on Control and Optimization 41 (2002), no. 4, 991-1014.

[4] A. Agrachev, M. Sigalotti, On the local structure of optimal trajectories in $\mathbb{R}^{3}$, SIAM J. on Control and Optimization 42, (2003), 513-531.

[5] A. Agrachev, Y. Sachkov, Control Theory from the Geometric Viewpoint, Encyclopaedia of Mathematical Sciences, 87, Control Theory and Optimization, II, Springer-Verlag, Berlin, 2004.

[6] V. I. Arnold, Mathematical Methods of Classical Mechanics, Graduate Texts in Marhematics, 60, Springer-Verlag, Berlin, 1989.

[7] J.-P. Aubin, H. Frankowska, Set-Valued Analysis, Birkhäuser, Boston, 1990.

[8] D. Augustin, H. Maurer, Computational sensitivity analysis for state constrained optimal control problems, Ann. Oper. Res. 101 (2001), pp. 75-99.

[9] G. A. Bliss, Lectures on the Calculus of Variations, The University of Chicago Press, Chicago, 1968.

[10] V. G. Boltyanskii, Sufficient conditions for optimality and the justification of the dynamic programming method, SIAM J. Control 4 (1966), 326-361.

[11] B. Bonnard, J.-B. Caillau, E. Trélat, Second order optimality conditions in the smooth case and applications in optimal control, ESAIM Control Optim. Calc. Var. 13, 2 (2007), 207-236.

[12] A. Bressan, A high order test for optimality of bang-bang controls, SIAM J. Control Optim. 23 (1985), pp. 38-48.

[13] P. Cannarsa, C. Sinestrari, Semiconcave Functions, HamiltonJacobi Equations, and Optimal Control, Progress in Nonlinear Differential Equations and their Applications, 58, Birkhäuser Boston, Inc., Boston, MA, 2004.

[14] L. Cesari, Optimization - Theory and Applications. Problems with Ordinary Differential Equations. Applications of Mathematics 17, Springer-Verlag, New York, 1983.

[15] F.H. Clarke, R. Vinter, The relationship between the maximum principle and dynamic programming, SIAM J. Control Optim., 25 (1987), no. 5, 1291-1311.

[16] J. C. Dunn, Second-order optimality conditions in sets of $L^{\infty}$ functions with range in a polyhedron, SIAM J. Control Optim. 33 (1995), 1603-1635.

[17] M. R. Hestenes, Application of the theory of quadratic forms in Hilbert spaces to the calculus of variations, Pac. J. Math. 1 (1951), 525-582.

[18] M. D. Kiefer, H. Schättler, Parametrized families of extremals and singularities in solutions to the Hamilton-Jacobi-Bellman equation, SIAM J. Control Optim. 37 (1999), pp. 1346-1371.

[19] A. J. Krener, H. Schättler, The structure of small-time reachable sets in low dimensions, SIAM J. Cont. Opt. 27, No. 1 (1989), pp. 120-147.

[20] U. Ledzewicz, H. Schättler, Optimal bang-bang controls for a two-compartment model in cancer chemotherapy, J. Optim. Theory Appl. 114 (2002), pp. 609-637.

[21] K. Malanowski, H. Maurer, Sensitivity analysis for parametric control problems with control-state constraints, Comput. Optim. Appl. 5 (1996), pp. 253-283.

[22] K. Malanowski, H. Maurer, Sensitivity analysis for state constrained optimal control problems, Discrete Contin. Dynam. Systems 4 (1998), pp. 241-272.

[23] K. Malanowski, H. Maurer, Sensitivity analysis for optimal control problems subject to higher order state constraints, Optimization with data perturbations, II, Ann. Oper. Res. 101 (2001), pp. 43-73.

[24] H. Maurer, First and second order sufficient optimality conditions in mathematical programming and optimal control, Mathematical Programming Study 14 (1981), 163-177. 
[25] H. Maurer, D. Augustin, Second order sufficient conditions and sensitivity analysis for the controlled Rayleigh problem, in Parametric Optimization and Related Topics, IV, J. Guddat et al., eds., Lang, Frankfurt (1997), pp. 245-259.

[26] H. Maurer, D. Augustin, Sensitivity analysis and real-time control of parametric optimal control problems using boundary value methods, in On-line Optimization of Large Scale Systems, M. Grötschel et al., eds., Springer-Verlag, Berlin (2001), pp. 1755.

[27] H. Maurer, C. Büskens, J.-H. R. Kim, C.Y. Kaya, Optimization methods for the verification of second order sufficient conditions for bang-bang controls, Optim. Control Appl. Meth. 26 (2005), 129-156.

[28] H. Maurer, H. J. Oberle, Second order sufficient conditions for optimal control problems with free final time: The Riccati approach, SIAM J. Control Optim. 41 (2002), pp. 380-403.

[29] H. Maurer, N.P. Osmolovskii, Second order optimality conditions for bang-bang control problems, Control and Cybernetics 32, No. 3 (2003), 555-584.

[30] H. Maurer, N.P. Osmolovskii, Second order sufficient conditions for time-optimal bang-bang control problems, SIAM Journal on Control and Optimization 42 (2004), 2239-2263.

[31] H. Maurer, S. Pickenhain, Second-order sufficient conditions for optimal control problems with mixed control-state constraints, $\mathrm{J}$. Optim. Theory Appl. 86 (1995), pp. 649-667.

[32] A. A. Milyutin, N. P. Osmolovskii, Calculus of Variations and Optimal Control, Transl. Math. Monogr. 180, AMS, Providence, RI (1998).

[33] J. Noble, H. Schättler, Sufficient conditions for relative minima of broken extremals in optimal control theory, J. Math. Anal. Appl. 269 (2002), 98-128.

[34] N.P. Osmolovskii. Second order conditions for broken extremal. In: Calculus of Variations and Optimal Control. (Technion 1998), A. loffe, S. Reich and I. Shafir, eds.. Chapman and Hall/CRC, Boca Raton, Horida, 198-216, 2000.

[35] N.P. Osmolovskii, H. Maurer, Equivalence of second order optimality conditions for bang-bang control problems. Part 1: main results, Control and Cybernetics 34 (2005), no. 3, 927-949.

[36] N.P. Osmolovskii, H. Maurer, Equivalence of second order optimality conditions for bang-bang control problems. Part 2: Proofs, variational derivatives and representations, Control and Cybernetics 36, 555-584.

[37] N.P. Osmolovskii, F. Lempio, Transformation of quadratic forms to perfect squares for broken extremal, Set-Valued Anal. 10 (2002), 209-232.

[38] L. Poggiolini, G. Stefani, State-local optimality of a bang-bang trajectory: a Hamiltonian apprach, Systems and Control Letters 53 (2004), 269-279.

[39] L. Pontryagin, V. Boltyanskii, R. Gramkrelidze, E. Mischenko, The Mathematical Theory of Optimal Processes, Wiley Interscience, 1962.

[40] L. Rifford, E. Trélat, Morse-Sard type results in sub-Riemannian geometry, Math. Ann. 332 (2005), no. 1, 145-159.

[41] L. Rifford, E. Trélat, On the stabilization problem for nonholonomic distributions, J. Eur. Math. Soc., 11 (2009), no. 2, $223-$ 255.

[42] A. V. Sarychev, The index of second variation of a control system, Matem. Sbornik 113 (1980), 464-486. English transl. in: Math. USSR Sbornik 41 (1982), 383-401.

[43] A. V. Sarychev, First- and second-order sufficient optimality conditions for bang-bang controls, SIAM J. Control Optim. 35 (1997), 315-440.

[44] C. J. Silva, E. Trélat, Smooth regularization of bang-bang optimal control problems, accepted for publication in IEEE - Transactions on Automatic Control (2009).
[45] G. Stefani, Regularity properties of the minimum-time map, Nonlinear synthesis (Sopron, 1989), 270-282, Progr. Systems Control Theory, 9, Birkhäuser Boston, Boston, MA, 1991.

[46] H.J. Sussmann, Envelopes, conjugate points and optimal bangbang extremals, in Proc. 1985 Paris Conf. on Nonlinear Systems, M. Fliess and M. Hazewinkel, eds., Reidel Publishers, Dordrecht, the Netherlands (1987).

[47] H.J. Sussmann, B. Piccoli, Regular synthesis and sufficient conditions for optimality, SIAM J. Control Optim. 39 (2) (2000), pp. $359-410$.

[48] G. Vossen, H. Maurer, On $L^{1}$-minimization in optimal control and applications to robotics, Optim. Control Appl. Meth. 27 (2006), pp. 301-321.

[49] V. Zeidan, The Riccati equation for optimal control problems with mixed state-control constraints: Necessity and sufficiency, SIAM J. Control Optim. 32 (1994), pp. 1297-1321. 